\title{
Synthesis of Novel Functionalized 3-spiropyrrolizidine and 3-spiropyrrolidine oxindoles from Baylis-Hillman adducts of Isatin and Heteroaldehydes with Azomethine Ylides via [3+2]-cycloaddition
}

\author{
Ponnusamy Shanmugam*, Baby Viswambharan and Suchithra Madhavan \\ Chemical Sciences and Technology Division, National Institute for Interdisciplinary Science and \\ Technology (NIST), Trivandrum-695 019, Kerala, India \\ E-mail: shanmu196@rediffmail.com
}

\section{$\underline{\text { Contents }}$}

(1) General remarks

(2) General experimental procedure

(3) Spectral characterization of new compounds

(4) Scanned ${ }^{1} \mathrm{H}$ and ${ }^{13} \mathrm{C}$ NMR's and FAB mass spectra

\section{(1) General Considerations}

All the reactions were carried out in oven-dried glassware. Progress of reactions was monitored by Thin Layer Chromatography (TLC) while purification of crude compounds was done by column chromatography using silica gel (100-200 mesh). NMR spectra were recorded at 300.1 MHz $\left({ }^{1} \mathrm{H}\right)$ and 75.3 $\mathrm{MHz}\left({ }^{13} \mathrm{C}\right)$ respectively on a Brucker Advance DPX-300 spectrometer. Chemical shifts are reported in $\delta(\mathrm{ppm})$ relative to TMS $\left({ }^{1} \mathrm{H}\right)$ or $\mathrm{CDCl}_{3}\left({ }^{13} \mathrm{C}\right)$ as internal standards. IR spectra were recorded on Bomem MB series FT-IR spectrometer; absorbencies are reported in $\mathrm{cm}^{-1}$. FAB mass /HRMS were measured at the JMS 600 JEOL Mass Spectrometer. Yields refer to quantities obtained after chromatography.

\section{(2) General experimental procedure:}

A mixture of Baylis-Hillman adducts (100 mg, $0.404 \mathrm{mmol}), \mathrm{L}-(-)$ proline or sarcosine (1.2eq), isatin (1.2eq.) and montmorillonite $\mathrm{K}-10$ Clay $(100 \% \mathrm{w} / \mathrm{w})$ in methanol $(1 \mathrm{~mL})$ was refluxed for 0.5-6 hours. After the reaction (TLC), the crude mixture was filtered through a pad of celite and then purified by a silica gel column chromatography to afford products in very good yields $(68-75 \%)$.

\section{(3) Characterization data}




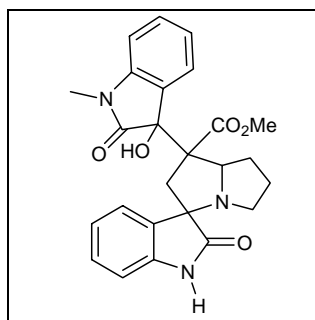

Compound $\mathbf{8}$

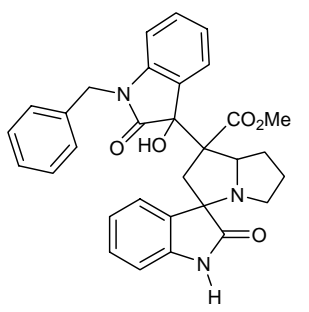

Compound 9

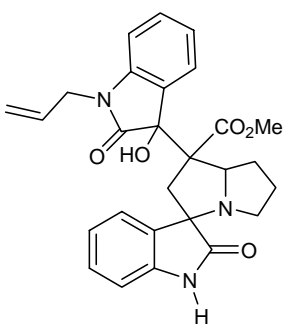

Compound $\mathbf{1 0}$
IR $\left(\mathrm{CH}_{2} \mathrm{Cl}_{2}\right): y_{\max }: 753,1091,1347.00,1470,1614,1723,2950,3272 \mathrm{~cm}^{-1}$; ${ }^{1} \mathbf{H} \mathbf{N M R}\left(\mathrm{CDCl}_{3} / \mathrm{TMS}, 300.1 \mathrm{MHz}\right): \delta 1.34-1.47(\mathrm{~m}, 1 \mathrm{H}), 1.97-2.07(\mathrm{~m}, 2 \mathrm{H})$, 2.13-2.43(m, 1H), 2.29-2.37(dd, $1 \mathrm{H}, J=8.2 \mathrm{~Hz}, J=15 \mathrm{~Hz}), 2.56-2.86(\mathrm{dd}, 1 \mathrm{H}$, $J=8.1 \mathrm{~Hz}, J=13.1 \mathrm{~Hz}), 3.20(\mathrm{~s}, 3 \mathrm{H}), 3.21-3.29(\mathrm{~d}, 1 \mathrm{H}, J=15 \mathrm{~Hz}), 3.46(\mathrm{~s}, 3 \mathrm{H})$, $3.49-3.54(\mathrm{~d}, 1 \mathrm{H}, J=15 \mathrm{~Hz}), 4.87-4.92(\mathrm{dd}, 1 \mathrm{H}, J=6.3 \mathrm{~Hz}, J=8.4 \mathrm{~Hz}), 5.65(\mathrm{~s}$, $1 \mathrm{H}), 6.67-6.78(\mathrm{~d}, 1 \mathrm{H}, J=6 \mathrm{~Hz}), 6.85-6.88(\mathrm{~d}, 1 \mathrm{H}, J=9 \mathrm{~Hz}), 6.95-7.00(\mathrm{t}, 1 \mathrm{H}$, $J=9 \mathrm{~Hz}), \quad 7.05-7.01(\mathrm{t}, 1 \mathrm{H}, J=6 \mathrm{~Hz}), 7.21-7.30(\mathrm{~m}, 3 \mathrm{H}), 7.47-7.50(\mathrm{~d}, 1 \mathrm{H}$, $J=9 \mathrm{~Hz}), 8.37(\mathrm{~s}, \mathrm{H}) ;{ }^{13} \mathbf{C ~ N M R}\left(\mathrm{CDCl}_{3} / \mathrm{TMS}, 75.3 \mathrm{MHz}\right): \delta 26.11,26.75$, $42.60,43.00,52.03,60.68,65.84,68.15,75.63,107.96,110.28,122.65$, $123.08,123.85,124.47,125.30,128.95,129.23,129.61,129.97,140.93$, 144.28, 172.45, 176.81, 182.25. FAB mass: Calcd. for $\mathrm{C}_{25} \mathrm{H}_{25} \mathrm{~N}_{3} \mathrm{O}_{5}$ : 447.48; Found: 448.65(M+1).

IR( $\left(\mathrm{CH}_{2} \mathrm{Cl}_{2}\right): y_{\max }: 754,1034,1113,1465,1610,1732,2919,3351.64 \mathrm{~cm}^{-1}$; ${ }^{1} \mathbf{H}$ NMR(CDCl ${ }_{3}$-DMSO(3:1)/TMS, 300.1 MHz): $\delta 1.33-1.46(\mathrm{~m}, 1 \mathrm{H}), 1.93-$ $2.00(\mathrm{~m}, 2 \mathrm{H}), 2.15-2.21(\mathrm{~m}, 1 \mathrm{H}), 2.30-2.38(\mathrm{dd}, 1 \mathrm{H}, J=8.1 \mathrm{~Hz}, J=14.4 \mathrm{~Hz})$, 2.63-2.71(m, $1 \mathrm{H}), 3.11(\mathrm{~s}, 3 \mathrm{H}), 3.36-3.41(\mathrm{~d}, 1 \mathrm{H}, J=15 \mathrm{~Hz}), 3.54-3.59(\mathrm{~d}, 1 \mathrm{H}$, $J=15 \mathrm{~Hz}), 4.76-4.81(\mathrm{~d}, 1 \mathrm{H}, J=15 \mathrm{~Hz}), 4.93-5.03(\mathrm{~m}, 2 \mathrm{H}), 6.30(\mathrm{~s}, 1 \mathrm{H}), 6.73-$ 6.76(d, 1H, J=9Hz), 6.87-6.91(m, 2H), 7.01-7.06(t, $1 \mathrm{H}, J=6 \mathrm{~Hz}), 7.14-$ $7.36(\mathrm{~m}, \quad 6 \mathrm{H}), \quad 7.43-7.45(\mathrm{~m}, \quad 3 \mathrm{H}), \quad 10.04(\mathrm{~s}, \quad 1 \mathrm{H}) ;{ }^{13} \mathbf{C} \quad \mathbf{N M R} \quad\left(\mathrm{CDCl}_{3^{-}}\right.$ DMSO(3:1)/TMS, 75.3 MHz): $\delta$ 25.40, 26.40, 42.07, 42.53, 43.38, 51.23, $60.70,65.55,67.55,72.09,74.55,76.57,108.29,109.80,111.13,122.05$, $122.09,123.61,124.69,127.22,127.56,128.26,128.69,129.05,129.22$, 135.71, 141.46, 142.94, 172.07, 176.55, 181.53. FAB mass: Calcd. for $\mathrm{C}_{31} \mathrm{H}_{29} \mathrm{~N}_{3} \mathrm{O}_{5}$ : 523.21; Found: 524.83(M+1).

IR $\left(\mathrm{CH}_{2} \mathrm{Cl}_{2}\right): y_{\max }: 753.68,1222.59,1359,1459,1469,1614,1723,2951$, $3272.01 \mathrm{~cm}^{-1}$; ${ }^{1} \mathbf{H} \mathbf{N M R}\left(\mathrm{CDCl}_{3} / \mathrm{TMS}, 300.1 \mathrm{MHz}\right): \delta 1.35-1.47(\mathrm{~m}, 1 \mathrm{H}), 1.94-$ $1.99(\mathrm{~m}, 2 \mathrm{H}), 2.07-2.19(\mathrm{~m}, 1 \mathrm{H}), 2.19-2.366(\mathrm{dd}, 1 \mathrm{H}, J=15 \mathrm{~Hz}, J=8.1 \mathrm{~Hz}), 2.56-$ 2.63(dd, $1 \mathrm{H}, J=8.1 \mathrm{~Hz}, J=13.2 \mathrm{~Hz}), 3.27-3.317(\mathrm{~d}, 1 \mathrm{H}, J=14.1 \mathrm{~Hz}), 3.46(\mathrm{~S}$, $3 \mathrm{H}), \quad 3.505-3.552(\mathrm{~d}, \quad 1 \mathrm{H}, \quad J=14.1 \mathrm{~Hz}), \quad 4.193-4.266(\mathrm{dd}, \quad 1 \mathrm{H}, \quad J=5.7 \mathrm{~Hz}$, $J=15.9 \mathrm{~Hz}$ ), 4.378-4.449(dd, $1 \mathrm{H}, J=5.1 \mathrm{~Hz}, J=15.9 \mathrm{~Hz}$ ), 4.897-4.894(dd, $1 \mathrm{H}$, $J=6 \mathrm{~Hz}, \quad J=8.4 \mathrm{~Hz}), \quad 5.233-5.20(\mathrm{dd}, \quad 1 \mathrm{H}, \quad J=0.9 \mathrm{~Hz}, \quad J=10.3 \mathrm{~Hz}), \quad 5.328$ $5.388(\mathrm{dd}, 1 \mathrm{H}, J=0.9 \mathrm{~Hz}, J=17.1 \mathrm{~Hz}), 5.695(\mathrm{bs}, 1 \mathrm{H}), 5.803-5.931(\mathrm{~m}, 1 \mathrm{H})$, 6.772-6.798(d, 1H, J=7.8 Hz), 6.868-6.893(d, 1H, J=7.5 Hz), 6.930- 


\begin{tabular}{|c|c|}
\hline & $\begin{array}{l}\text { 6.980(t, } 1 \mathrm{H}, J=7.5 \mathrm{~Hz}), 7.047-7.095(\mathrm{t}, 1 \mathrm{H}, J=6.9 \mathrm{~Hz}), 7.234-7.277(\mathrm{~m}, 3 \mathrm{H}), \\
\text { 7.476-7.50(d, } 1 \mathrm{H}, J=7.2 \mathrm{~Hz}), 8.813(\mathrm{~S}, 1 \mathrm{H}) .{ }^{13} \mathbf{C} \mathbf{N M R}\left(\mathrm{CDCl}_{3} / \mathrm{TMS}, 75.3\right. \\
\mathrm{MHz}): \delta 26.06,26.65,42.48(2 \mathrm{C}), 43.03,51.93,56.01,65.78,68.17,75.42, \\
\text { 76.57, 108.757, 110.19, 118.08, 122.54, 123.05, 124.49, 125.301, 129.19, } \\
\text { 129.59, 129.76, 131.56, 140.88, 143.52, 178.45, 176.43, 182.08. FAB mass: } \\
\text { Calcd. for } \mathrm{C}_{27} \mathrm{H}_{27} \mathrm{~N}_{3} \mathrm{O}_{5}: 473.1951 \text {; Found: 474.69(M+1). }\end{array}$ \\
\hline Com & $\begin{array}{l}{ }^{1} \mathbf{H} \text { NMR }\left(\mathrm{CDCl}_{3} / \mathrm{TMS}, 300.1 \mathrm{MHz}\right): \delta 1.42(\mathrm{~m}, 1 \mathrm{H}), 2.08-2.13(\mathrm{~m}, 2 \mathrm{H}) \text {, } \\
2.15-2.18(\mathrm{~m}, 1 \mathrm{H}), 2.31-2.33(\mathrm{~m}, 1 \mathrm{H}), 2.27-2.79(\mathrm{~m}, 2 \mathrm{H}), 3.25(\mathrm{~s}, 3 \mathrm{H}), 3.48- \\
\text { 3.64(two doublets, } J=15 \mathrm{~Hz}, 2 \mathrm{H}), 5.23(\mathrm{bs}, 1 \mathrm{H}), 6.33-6.35(\mathrm{~m}, 1 \mathrm{H}), 6.51(\mathrm{~m} \text {, } \\
1 \mathrm{H}), 6.77-6.79(\mathrm{~d}, 1 \mathrm{H}, J=6 \mathrm{~Hz}), 6.91-6.93(\mathrm{~d}, 1 \mathrm{H}, J=6 \mathrm{~Hz}), 7.09-7.35(\mathrm{~m}, 8 \mathrm{H}) \text {, } \\
\text { 7.65-7.68(d, } J=3 \mathrm{~Hz}, 1 \mathrm{H}), 8.4(\mathrm{~s}, 1 \mathrm{H}) ; \mathbf{F A B} \text { mass: Calcd. for } \mathrm{C}_{29} \mathrm{H}_{27} \mathrm{~N}_{3} \mathrm{O}_{5} \mathrm{~S} \text { : } \\
\text { 529.17; Found: } 530.12(\mathrm{M}+1) .\end{array}$ \\
\hline & 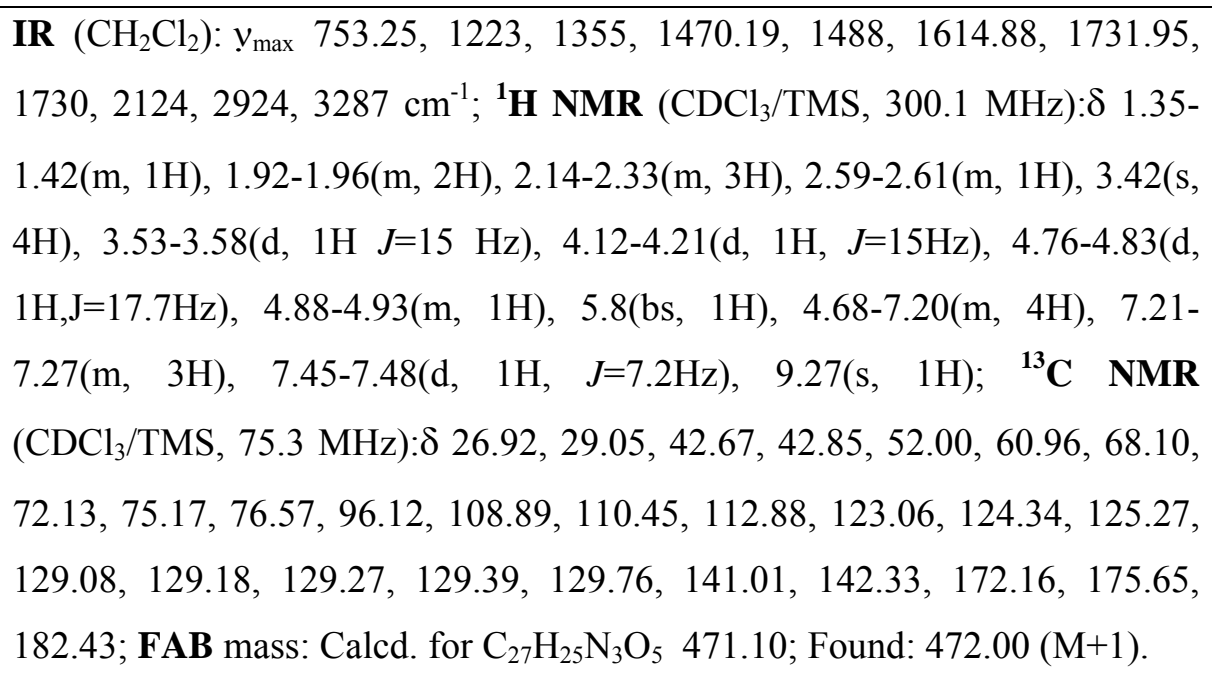 \\
\hline & $\begin{array}{l}{ }^{1} \mathbf{H} \text { NMR }\left(\mathrm{CDCl}_{3} / \mathrm{TMS}, 300.1 \mathrm{MHz}\right): \delta 1.63-1.70(\mathrm{~m}, 1 \mathrm{H}), 1.95-1.99(\mathrm{~m}, 2 \mathrm{H}) \text {, } \\
2.28(\mathrm{~m}, 5 \mathrm{H}), 2.49-2.52(\mathrm{~m}, 1 \mathrm{H}), 2.65-2.68(\mathrm{~m}, 1 \mathrm{H}), 2.83-2.91(\mathrm{~m}, 1 \mathrm{H}), 3.05- \\
3.12(\mathrm{~m}, 1 \mathrm{H}), 4.14-4.16(\mathrm{~m}, 1 \mathrm{H}), 6.96-7.07(\mathrm{~m}, 2 \mathrm{H}), 7.18-7.29(\mathrm{~m}, 2 \mathrm{H}), 9.3(\mathrm{~s}, \\
1 \mathrm{H}) \text {; FAB mass: Calcd. for } \mathrm{C}_{16} \mathrm{H}_{18} \mathrm{~N}_{2} \mathrm{O}_{5} \text { 270.32; Found: } 271.41(\mathrm{M}+1) \text {. }\end{array}$ \\
\hline Compound 18 & $\begin{array}{l}\text { IR }\left(\mathrm{CH}_{2} \mathrm{Cl}_{2}\right): y_{\max }: 753,1091,1347.00,1470,1614,1723,2950,3272 \mathrm{~cm}^{-1} ; \\
{ }^{1} \mathbf{H} \mathbf{N M R}\left(\mathrm{CDCl}_{3} / \mathrm{TMS}, 300.1 \mathrm{MHz}\right): \delta 1.75(\mathrm{brs}, 1 \mathrm{H}), 1.87(\mathrm{~s}, 3 \mathrm{H}), 2.98, \\
3.00(\mathrm{~s}, 6 \mathrm{H}), 3.17-3.22(\mathrm{~m}, 3 \mathrm{H}), 3.36(\mathrm{~s}, 4 \mathrm{H}), 6.64-6.66(\mathrm{~d}, 1 \mathrm{H}, J=6 \mathrm{~Hz}), 6.80- \\
6.86(\mathrm{~m}, 3 \mathrm{H}), 6.93-6.98(\mathrm{~m}, 2 \mathrm{H}), 7.20-7.30(\mathrm{~m}, 2 \mathrm{H}) ;{ }^{13} \mathbf{C ~ N M R}\left(\mathrm{CDCl}_{3} / \mathrm{TMS},\right. \\
75.3 \mathrm{MHz}): \delta 25.98,26.40,28.56(2 \mathrm{C}), 34.24(2 \mathrm{C}), 51.31,51.44,67.19, \\
107.58,108.39(2 \mathrm{C}), 122.31,122.50,124.63,127.61(2 \mathrm{C}), 129.22,129.70, \\
143.95,144.21,171.11,175.98,178.16 ; \mathbf{F A B} \text { mass: Calcd. for } \mathrm{C}_{24} \mathrm{H}_{25} \mathrm{~N}_{3} \mathrm{O}_{5} \text { : }\end{array}$ \\
\hline
\end{tabular}




\begin{tabular}{|c|c|}
\hline & 435.47; Found: $435.97\left(\mathrm{M}^{+}\right)$ \\
\hline Cor & $\begin{array}{l}\text { IR( }\left(\mathrm{CH}_{2} \mathrm{Cl}_{2}\right): y_{\max }: 754,1034,1113,1465,1610,1732,2919,3351 \mathrm{~cm}^{-1} ;{ }^{1} \mathbf{H} \\
\text { NMR }\left(\mathrm{CDCl}_{3} / \mathrm{TMS}, 300.1 \mathrm{MHz}\right): \delta 2.04(\mathrm{~s}, 3 \mathrm{H}), 2.55(\mathrm{~s}, 3 \mathrm{H}), 3.19-3.34(\mathrm{~m} \text {, } \\
4 \mathrm{H}), 4.44-4.49(\mathrm{~d}, 1 \mathrm{H}, J=15 \mathrm{~Hz}), 4.82-4.89(\mathrm{~d}, 1 \mathrm{H}, J=15 \mathrm{~Hz}), 6.45(\mathrm{~s}, 1 \mathrm{H}), 6.68- \\
6.75(\mathrm{~m}, 2 \mathrm{H}), 6.92-7.00(\mathrm{~m}, 3 \mathrm{H}), 7.09-7.26(\mathrm{~m}, 6 \mathrm{H}), 7.85-7.31(\mathrm{~m}, 2 \mathrm{H}), 8.02(\mathrm{~s}, \\
1 \mathrm{H}) \text {; FAB mass: Calcd. for } \mathrm{C}_{29} \mathrm{H}_{27} \mathrm{~N}_{3} \mathrm{O}_{5} 497.54 \text {; Found: } 497.60\left(\mathrm{M}^{+}\right)\end{array}$ \\
\hline Comp & $\begin{array}{l}\text { IR }\left(\mathrm{CH}_{2} \mathrm{Cl}_{2}\right): v_{\max }: 751.30,1102,1366,1467,1612,1676,1712,2923,3269 \\
\mathrm{~cm}^{-1} ;{ }^{1} \mathbf{H} \mathbf{N M R}\left(\mathrm{CDCl}_{3} / \mathrm{TMS}, 300.1 \mathrm{MHz}\right): \delta 1.71(\mathrm{brs}, 1 \mathrm{H}), 2.04(\mathrm{~s}, 3 \mathrm{H}), \\
3.06(\mathrm{~s}, 3 \mathrm{H}), 3.24-3.32(\mathrm{~m}, 4 \mathrm{H}), 4.05-4.13(\mathrm{dd}, 1 \mathrm{H}, J=6 \mathrm{~Hz}, J=18 \mathrm{~Hz}), 4015- \\
4.22(\mathrm{dd}, 1 \mathrm{H}, J=6 \mathrm{~Hz}, J=15 \mathrm{~Hz}), 5.10-5.13(\mathrm{~d}, 1 \mathrm{H}, J=10.2 \mathrm{~Hz}), 5.17-5.23(\mathrm{~d}, 1 \mathrm{H}, \\
J=15.9 \mathrm{~Hz}), 5.60-5.73(\mathrm{~m}, 1 \mathrm{H}), 6.69-6.72(\mathrm{~d}, 1 \mathrm{H}, J=9 \mathrm{~Hz}), 6.78-7.01(\mathrm{~m}, 5 \mathrm{H}), \\
7.17-7.21(\mathrm{~m}, 1 \mathrm{H}), 7.47(\mathrm{~s}, 1 \mathrm{H}), 8.55(\mathrm{~s}, 1 \mathrm{H}) ;{ }^{13} \mathrm{C} \mathbf{N M R}\left(\mathrm{CDCl}_{3} / \mathrm{TMS}, 75.3\right. \\
\mathrm{MHz}): \delta 28.48,34.31(2 \mathrm{C}), 42.30,51.06(2 \mathrm{C}), 51.53,66.91,108.61,110.29, \\
118.00,122.16,122.78(2 \mathrm{C}), 125.01,128.03,129.28,129.69,129.92,131.13, \\
141.55,143.09,171.14,172.14,179.81 ; \mathbf{F A B} \text { mass: Calcd. for } \mathrm{C}_{25} \mathrm{H}_{25} \mathrm{~N}_{3} \mathrm{O}_{5} \text { : } \\
447.48 ; \text { Found : 448.31(M+1). }\end{array}$ \\
\hline & $\begin{array}{l}\text { IR }\left(\mathrm{CH}_{2} \mathrm{Cl}_{2}\right): v_{\max }: 751.30,1102,1366,1467,1612,1676,1712.86,2923, \\
3269 \mathrm{~cm}^{-1} ;{ }^{1} \mathbf{H} \text { NMR }\left(\mathrm{CDCl}_{3} / \mathrm{TMS}, 300.1 \mathrm{MHz}\right): \delta 1.74(\mathrm{brs}, 1 \mathrm{H}), 2.02(\mathrm{~s}, \\
3 \mathrm{H}), 3.05-3.10(\mathrm{~d}, 1 \mathrm{H}, J=15 \mathrm{~Hz}), 3.27-3.31(\mathrm{~d}, 1 \mathrm{H}, J=14.7 \mathrm{~Hz}) 3.4(\mathrm{~s}, 3 \mathrm{H}), 3.56- \\
3.60(\mathrm{~d}, 1 \mathrm{H}, J=12 \mathrm{~Hz}), 4.17-4.24(\mathrm{dd}, 1 \mathrm{H}, J=6 \mathrm{~Hz}, J=15 \mathrm{~Hz}), 4.35-4.42(\mathrm{~m}, 2 \mathrm{H}), \\
5.19-5.36(\mathrm{~m}, 2 \mathrm{H}), 5.79-5.88(\mathrm{~m}, 1 \mathrm{H}), 6.75-6.78(\mathrm{~d}, 1 \mathrm{H}, J=9 \mathrm{~Hz}), 6.81-684(\mathrm{~d} \text {, } \\
1 \mathrm{H}, J=9 \mathrm{~Hz}), 6.92-6.97(\mathrm{t}, 1 \mathrm{H}, J=9 \mathrm{~Hz}), 7.04-7.69(\mathrm{t}, 1 \mathrm{H}, J=9 \mathrm{~Hz}), 7.20-7.25(\mathrm{~m}, \\
3 \mathrm{H}), 7.33-7.36(\mathrm{~d}, 1 \mathrm{H}, J=9 \mathrm{~Hz}), 8.3(\mathrm{~s}, 1 \mathrm{H}) ; \mathbf{F A B} \text { mass: Calcd. for } \mathrm{C}_{25} \mathrm{H}_{25} \mathrm{~N}_{3} \mathrm{O}_{5} \text { : } \\
447.4831 \text {; Found: } 448.42(\mathrm{M}+1) .\end{array}$ \\
\hline Compound 22 & 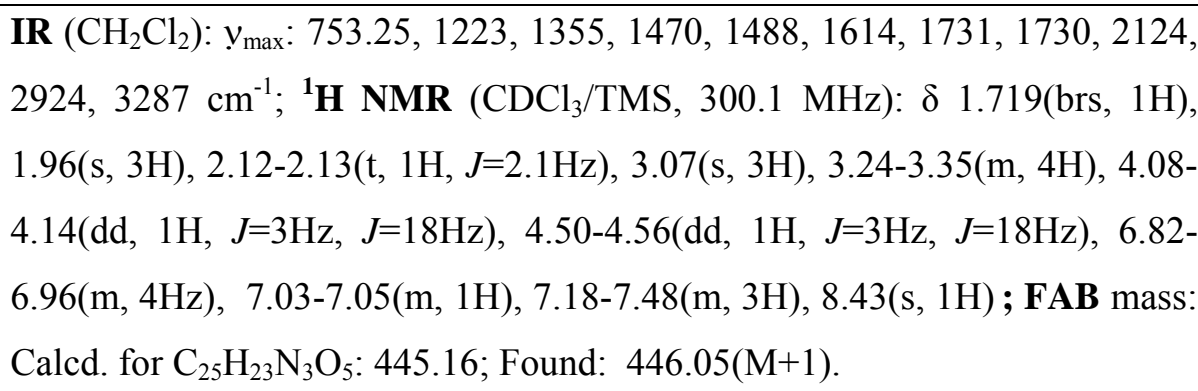 \\
\hline
\end{tabular}




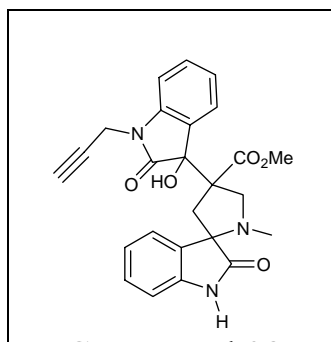

Compound 23

IR $\left(\mathrm{CH}_{2} \mathrm{Cl}_{2}\right): v \cdot \max 753.25,1223,1355,1470.19,1488,1614.88,1731.95$, 1730, 2124, 2924, 3287 $\mathrm{cm}^{-1} ;{ }^{1} \mathbf{H}$ NMR (CDCl $\left.3 / \mathrm{TMS}, 300.1 \mathrm{MHz}\right): \delta 2.14(\mathrm{~s}$, $3 \mathrm{H}), 2.25-2.27(\mathrm{t}, 1 \mathrm{H}, J=3 \mathrm{~Hz}), 3.12-3.17(\mathrm{~d}, 1 \mathrm{H}, J=14.1 \mathrm{~Hz}), 3.34-3.38(\mathrm{~d}, 1 \mathrm{H}$, $J=13.8 \mathrm{~Hz}), 3.45(\mathrm{~s}, 3 \mathrm{H}), 3.57-3.61(\mathrm{~d}, 1 \mathrm{H}, J=12 \mathrm{~Hz}), 4.17-4.24(\mathrm{dd}, 1 \mathrm{H}, J=3 \mathrm{~Hz}$, $J=18 \mathrm{~Hz}), 4.38-4.42(\mathrm{~d}, 1 \mathrm{H}, J=12 \mathrm{~Hz}), 4.76-4.82(\mathrm{dd}, 1 \mathrm{H}, J=3 \mathrm{~Hz}, J=18 \mathrm{~Hz})$, 5.42(brs, $1 \mathrm{H}), 6.87-6.89(\mathrm{~d}, 1 \mathrm{H}, J=6 \mathrm{~Hz}), 6.99-7.00(\mathrm{~m}, 2 \mathrm{H}), 7.10-7.12(\mathrm{~m}, 2 \mathrm{H})$, 7.23-7.40(m, $3 \mathrm{H}), 8.45(\mathrm{~s}, 1 \mathrm{H})$; FAB mass: Calcd. for $\mathrm{C}_{25} \mathrm{H}_{23} \mathrm{~N}_{3} \mathrm{O}_{5}$ : 445.16; Found: 446.05(M+1).

(n)

${ }^{1} \mathbf{H}$ NMR $\left(\mathrm{CDCl}_{3} / \mathrm{TMS}, 300.1 \mathrm{MHz}\right): \delta 1.78(\mathrm{brs}, 1 \mathrm{H}), 1.88(\mathrm{~s}, 3 \mathrm{H}), 2.68-$ 2.72(m, 1H), 2.92(s, 3H), 3.05(s, 3H), 3.20-3.28(m, 1H), 3.41-3.44(m, $1 \mathrm{H})$, 3.89-3.98(m, $1 \mathrm{H}), 6.32-6.34(\mathrm{~d}, 1 \mathrm{H}, J=6 \mathrm{~Hz}), 6.54-6.66(\mathrm{~m}, 2 \mathrm{H}), 7.01-7.44(\mathrm{~d}$, $4 \mathrm{H}, J=6 \mathrm{~Hz}) 7.72-7.74(\mathrm{~d}, 1 \mathrm{H}, J=6 \mathrm{~Hz}) ; \mathbf{F A B}$ mass: Calcd. for $\mathrm{C}_{23} \mathrm{H}_{22} \mathrm{~N}_{4} \mathrm{O}_{3}$ : Compound 24 402.16; Found:402.36( $\left.\mathrm{M}^{+}\right)$.

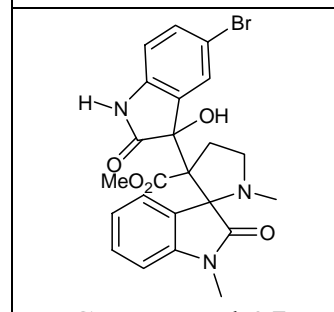

Compound 25

IR $\left(\mathrm{CH}_{2} \mathrm{Cl}_{2}\right): y_{\max }: 539,756,1237,1445,1471,1681,1732,2924,3282$, $1613 \mathrm{~cm}^{-1} ;{ }^{1} \mathbf{H}$ NMR (CDCl $\left.3 / \mathrm{TMS}, 300.1 \mathrm{MHz}\right): 1.80($ brs, $1 \mathrm{H}), 1.87(\mathrm{~s}, 3 \mathrm{H})$, $3.11(\mathrm{~s}, 3 \mathrm{H}), 3.14-3.24(\mathrm{~m}, 2 \mathrm{H}), 3.29(\mathrm{~s}, 5 \mathrm{H}), 6.50-6.52(\mathrm{~d}, 5 \mathrm{H}, J=6 \mathrm{~Hz}), 6.84-$ 6.87(m, 3H), 6.96-6.98(m, 1H), 7.01-7.35(m, 3H); FAB mass: Calcd. for $\mathrm{C}_{23} \mathrm{H}_{22} \mathrm{BrN}_{3} \mathrm{O}_{5}$ : 500.34; Found: 500.28( $\left.\mathrm{M}^{+}\right)$.

IR $\left(\mathrm{CH}_{2} \mathrm{Cl}_{2}\right): y_{\max }: 755,1337,1469,1519,1616,1738 \mathrm{~cm}^{-1} ;{ }^{1} \mathbf{H}$ NMR

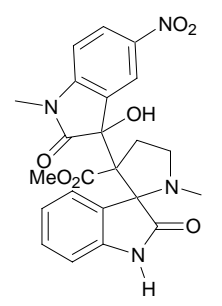
$\left(\mathrm{CDCl}_{3} / \mathrm{TMS}, 300.1 \mathrm{MHz}\right): \delta 2.10(\mathrm{~s}, 3 \mathrm{H}), 3.09-3.14(\mathrm{~d}, 1 \mathrm{H}, J=15 \mathrm{~Hz}), 3.14(\mathrm{~s}$, $3 \mathrm{H}), 3.30-3.35(\mathrm{~d}, 1 \mathrm{H}, J=15 \mathrm{~Hz}), 3.4(\mathrm{~s}, 3 \mathrm{H}), 3.38-3.48(\mathrm{~d}, 1 \mathrm{H}, J=12 \mathrm{~Hz}), 3.54-$ 3.58(d, 1H, $J=12 \mathrm{~Hz}), 5.9(\mathrm{brs}, 1 \mathrm{H}), 6.85-6.91(\mathrm{t}, 2 \mathrm{H}, J=9 \mathrm{~Hz}), 7.09-7.14(\mathrm{t}, 1 \mathrm{H}$, $J=6 \mathrm{~Hz}), 7.26-7.30(\mathrm{~m}, 1 \mathrm{H}), 7.30-7.34(\mathrm{~d}, 1 \mathrm{H}, J=9 \mathrm{~Hz}), 8.12(\mathrm{~s}, 1 \mathrm{H}), 8.26-$ 8.29(m, 2H); ${ }^{13} \mathrm{C}$ NMR ( $\left.\mathrm{CDCl}_{3} / \mathrm{TMS}, 75.3 \mathrm{MHz}\right): \delta$ 21.03, 26.54, 34.44, Compound 26 $37.87,53.02,57.88,59.33,70.71,74.91,107.67,110.16,120.57,123.77$, $125.02,127.13,129.23,129.23,129.61,140.73,143.28,149.63,172.82$, 176.98, 181.66; FAB mass: Calcd. for $\mathrm{C}_{23} \mathrm{H}_{22} \mathrm{~N}_{4} \mathrm{O}_{7}$ : 466.44; Found: 467.39(M+1).

IR $\left(\mathrm{CH}_{2} \mathrm{Cl}_{2}\right): v_{\max }: 1715,1745,3297 \mathrm{~cm}^{-1} ;{ }^{1} \mathbf{H} \mathbf{~ N M R}\left(\mathrm{CDCl}_{3} / \mathrm{TMS}, 300.1\right.$

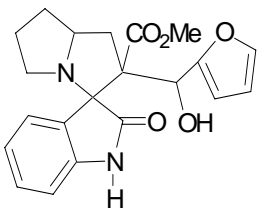
MHz): $\delta$ 1.44-1.48(m, 1H), 1.89(brs, 1H), 1.91-1.94(m, 2H), 2.04-2.09(m, $1 \mathrm{H}), 2.30-2.33(\mathrm{~m}, 1 \mathrm{H}), 2.44-2.49(\mathrm{~m}, 1 \mathrm{H}), 2.67-2.77(\mathrm{~m}, 2 \mathrm{H}), 3.07(\mathrm{~s}, 3 \mathrm{H})$, 4.26-4.28(m, 1H), 5.6(s, 1H), 6.28(s, 2H), 6.89-7.30(m, 5H), 8.8(s, $1 \mathrm{H}) ;{ }^{13} \mathrm{C}$ Compound $\mathbf{3 1}$ 


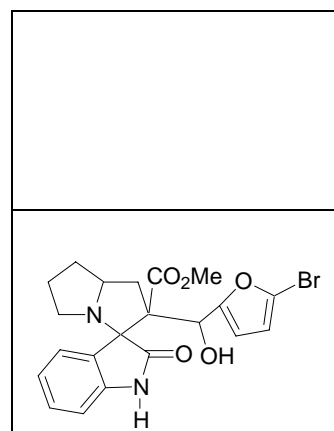

Compound 32

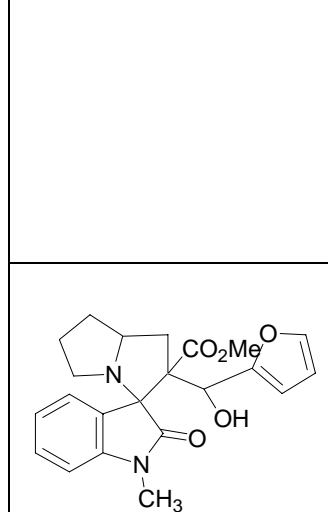

Compound 33

$67.2,72.3,108.6,110.4,110.8,123.5,127.3,128.4,130.5,140.7,143.3$, 152.8, 170.5, 180.3; FAB mass: Calcd. for $\mathrm{C}_{21} \mathrm{H}_{22} \mathrm{~N}_{2} \mathrm{O}_{5}$ : 382.41; Found: $383.40(\mathrm{M}+1)$.

IR $\left(\mathrm{CH}_{2} \mathrm{Cl}_{2}\right): v_{\max }: 690,1714,1744,3292 \mathrm{~cm}^{-1} ;{ }^{1} \mathbf{H} \mathbf{~ N M R}\left(\mathrm{CDCl}_{3} / \mathrm{TMS}\right.$, 300.1 MHz): $\delta$ 1.42-1.45(m, 1H), 1.91-1.96(m, 2H), 2.02-2.04(m, 1H), $2.30-$ 2.33(m, 1H), 2.46-2.49(m, $1 \mathrm{H}), 2.59-2.7(\mathrm{~m}, 2 \mathrm{H}), 3.12(\mathrm{~s}, 3 \mathrm{H}), 4.18-4.28(\mathrm{~m}$, $1 \mathrm{H}), 5.55(\mathrm{~s}, 1 \mathrm{H}), 6.19-6.20(\mathrm{~d}, 1 \mathrm{H}, \mathrm{J}=3), 6.30-6.31(\mathrm{~d}, 1 \mathrm{H}, \mathrm{J}=3)$ 6.9-7.26(m, 4H), 9.31(s, 1H); ${ }^{13} \mathbf{C}$ NMR $\left(\mathrm{CDCl}_{3} / \mathrm{TMS}, 75.3 \mathrm{MHz}\right): \delta$ 29.2, 33.9, 36.0, 50.7, 52.6, 54.5, 65.3, 67.2, 72.3, 96.1, 109.3, 110.9, 111.9, 121.0, 123.6, 127.2, 130.6, 140.9, 154.8, 170.3, 180.3; FAB mass: Calcd. for $\mathrm{C}_{21} \mathrm{H}_{21} \mathrm{BrN}_{2} \mathrm{O}_{5}$ : 461.31; Found: $461.46\left(\mathrm{M}^{+}\right)$

IR $\left(\mathrm{CH}_{2} \mathrm{Cl}_{2}\right): y_{\max }: 1712,1745,3435 \mathrm{~cm}^{-1} ;{ }^{1} \mathbf{H}$ NMR $\left(\mathrm{CDCl}_{3} / \mathrm{TMS}, 300.1\right.$ MHz): $\delta$ 1.42-1.47(m, 1H), 1.87-1.94(m, 2H ), 2.07-2.09(m, 1H), 2.212.24(m, 1H), 2.41-2.46(m, 1H), 2.61-2.75(m, 2H ), 3.01(s, 3H), 3.30(s, 3H), 4.21-4.31(m, 1H), 5.26(brs, $1 \mathrm{H}), 5.52(\mathrm{~s}, 1 \mathrm{H}), 6.26-6.27(\mathrm{~m}, 1 \mathrm{H}), 6.83-7.31(\mathrm{~m}$, 5H); ${ }^{13} \mathbf{C}$ NMR $\left(\mathrm{CDCl}_{3} / \mathrm{TMS}, 75.3 \mathrm{MHz}\right): \delta \quad 26.7,26.9,29.2,33.7,34.1$, 47.0, 51.4, 66.1, 71.2, 71.9, 107.8, 108.1, 109.9, 122.4, 125.8, 127.1, 129.4, 142.0, 143.8, 153.0, 171.2, 178.4; FAB mass: Calcd. for $\mathrm{C}_{22} \mathrm{H}_{24} \mathrm{~N}_{2} \mathrm{O}_{5}$ : 396.44; Found: $397.22(\mathrm{M}+1)$.

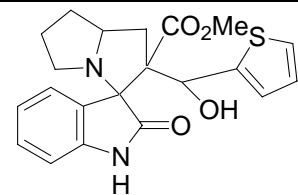

IR $\left(\mathrm{CH}_{2} \mathrm{Cl}_{2}\right): v$ max $_{\text {max }}: 1725,1745,3423 \mathrm{~cm}^{-1} ;{ }^{1} \mathbf{H}$ NMR $\left(\mathrm{CDCl}_{3} / \mathrm{TMS}, 300.1\right.$ MHz): $\delta$ 1.59-1.61(m, 1H), 1.91-1.93(m, 2H), 2.03-2.09(m, 2H), 2.262.43(m, 2H), 2.68-2.73(m, 2H), 3.08(s, 3H), 4.29(m, 1H ), 5.84(s, 1H), 6.74Compound 34 7.26(m, 7H), 7.97(s, $1 \mathrm{H})$; FAB mass: Calcd. for $\mathrm{C}_{21} \mathrm{H}_{22} \mathrm{~N}_{2} \mathrm{O}_{4} \mathrm{~S}$ : 398.48; Found: $398.78\left(\mathrm{M}^{+}\right)$.

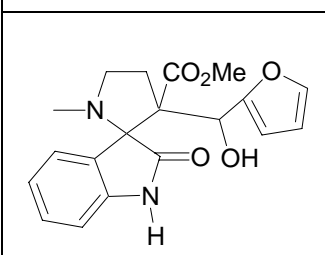

Compound $\mathbf{3 5}$
IR $\left(\mathrm{CH}_{2} \mathrm{Cl}_{2}\right): v$ max $_{\text {max }}$ 1715, 1729, $3340 \mathrm{~cm}^{-1} ;{ }^{1} \mathbf{H}$ NMR $\left(\mathrm{CDCl}_{3} / \mathrm{TMS}, 300.1\right.$ MHz): $\delta$ 2.04(s, 3H), 2.81-2.85(m, 2H), 2.98-3.0(m, 1H), 3.11(s, 3H ), 3.393.48(m, 1H), 5.72(s, $1 \mathrm{H}), 6.28-6.3(\mathrm{~m}, 2 \mathrm{H}), 6.92-7.28(\mathrm{~m}, 5 \mathrm{H}), 9.05(\mathrm{~s}, 1 \mathrm{H})$; ${ }^{13} \mathbf{C ~ N M R}\left(\mathrm{CDCl}_{3} / \mathrm{TMS}, 75.3 \mathrm{MHz}\right): \delta$ 25.1, 29.6, 50.5, 54.5, 65.6, 69.0, $70.5,108.8,109.4,111.2,123.5,125.5,126.9$, 127.0, 128.4, 140.7, 153.0, 170.4, 177.1; FAB mass: Calcd. for $\mathrm{C}_{19} \mathrm{H}_{20} \mathrm{~N}_{2} \mathrm{O}_{5}$ : 356.37; Found:357.23 $(\mathrm{M}+1)$.

IR $\left(\mathrm{CH}_{2} \mathrm{Cl}_{2}\right): y_{\max }: 669,1715,1749,3257 . \mathrm{cm}^{-1} ;{ }^{1} \mathbf{H} \mathbf{~ N M R}\left(\mathrm{CDCl}_{3} / \mathrm{TMS}\right.$, $300.1 \mathrm{MHz}): \delta \quad 2.05(\mathrm{~s}, 3 \mathrm{H}), 2.72-2.83(\mathrm{~m}, 2 \mathrm{H}), 2.95-3.02(\mathrm{~m}, 1 \mathrm{H}), 3.17(\mathrm{~s}$, 


\begin{tabular}{|l|l|}
$3 \mathrm{H}), 3.40-3.46(\mathrm{~m}, 1 \mathrm{H}), 5.33(\mathrm{bs}, 1 \mathrm{H}), 5.52(\mathrm{~s}, 1 \mathrm{H}), 6.36-6.37(\mathrm{~d}, 1 \mathrm{H}, J=3 \mathrm{~Hz})$, \\
$6.23-6.24(\mathrm{~d}, 1 \mathrm{H}, J=3 \mathrm{~Hz}), 6.95-7.27(\mathrm{~s}, 4 \mathrm{H}), 9.24(\mathrm{~s}, 1 \mathrm{H}) ; \mathbf{F A B}$ mass: Calcd. \\
for $\mathrm{C}_{19} \mathrm{H}_{19} \mathrm{BrN}_{2} \mathrm{O}_{5}: 434.05 ;$ Found: $434.00\left(\mathrm{M}^{+}\right)$.
\end{tabular}




\section{${ }^{1} \mathrm{H}$ NMR of compound 8}

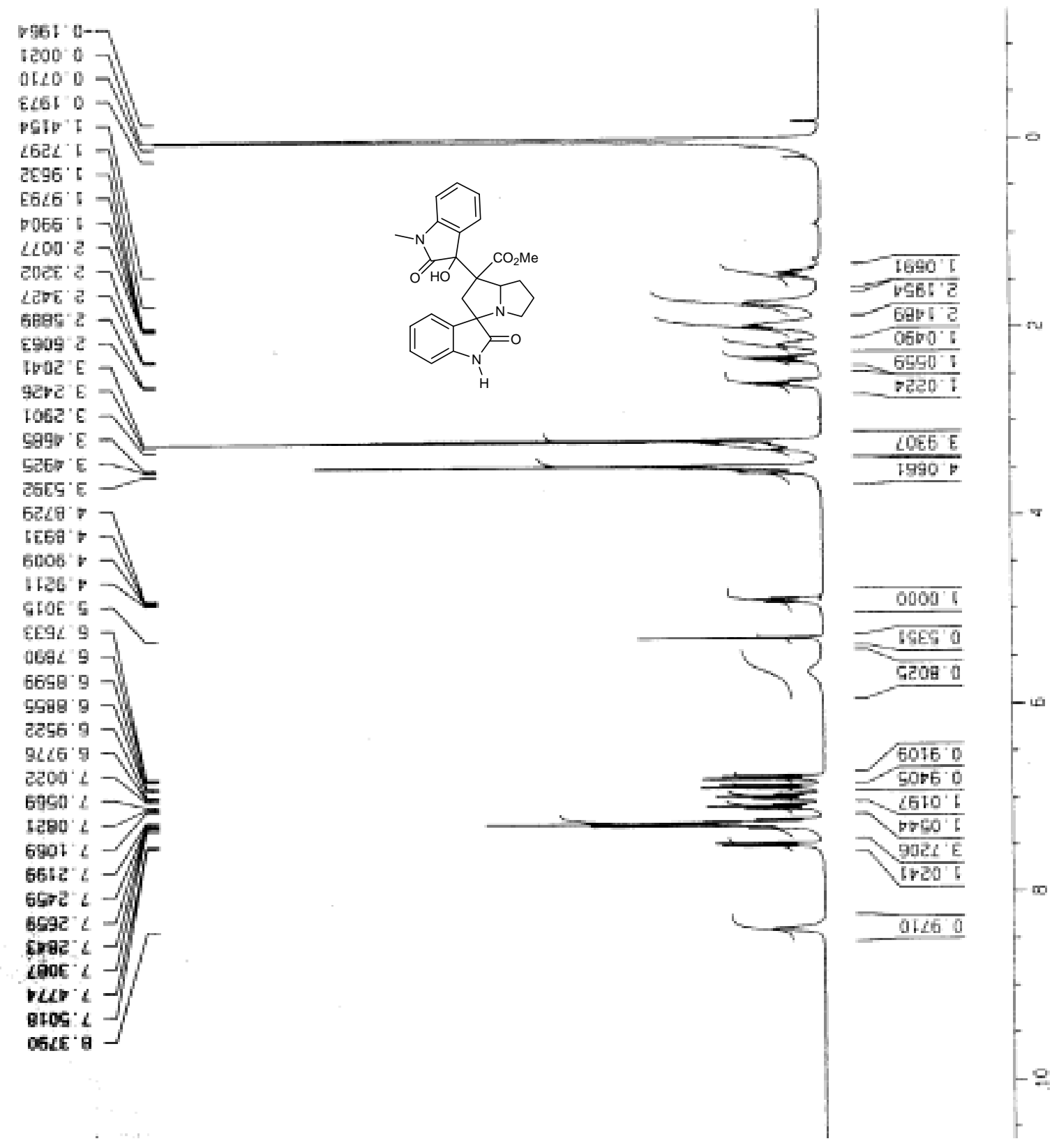




\section{${ }^{13}$ C NMR spectra of 8}

$2000-$


ESD $2 L 5-$

ह58 945

$05 \mathrm{C} 2 \mathrm{~s}-$

FAB mass of 8 

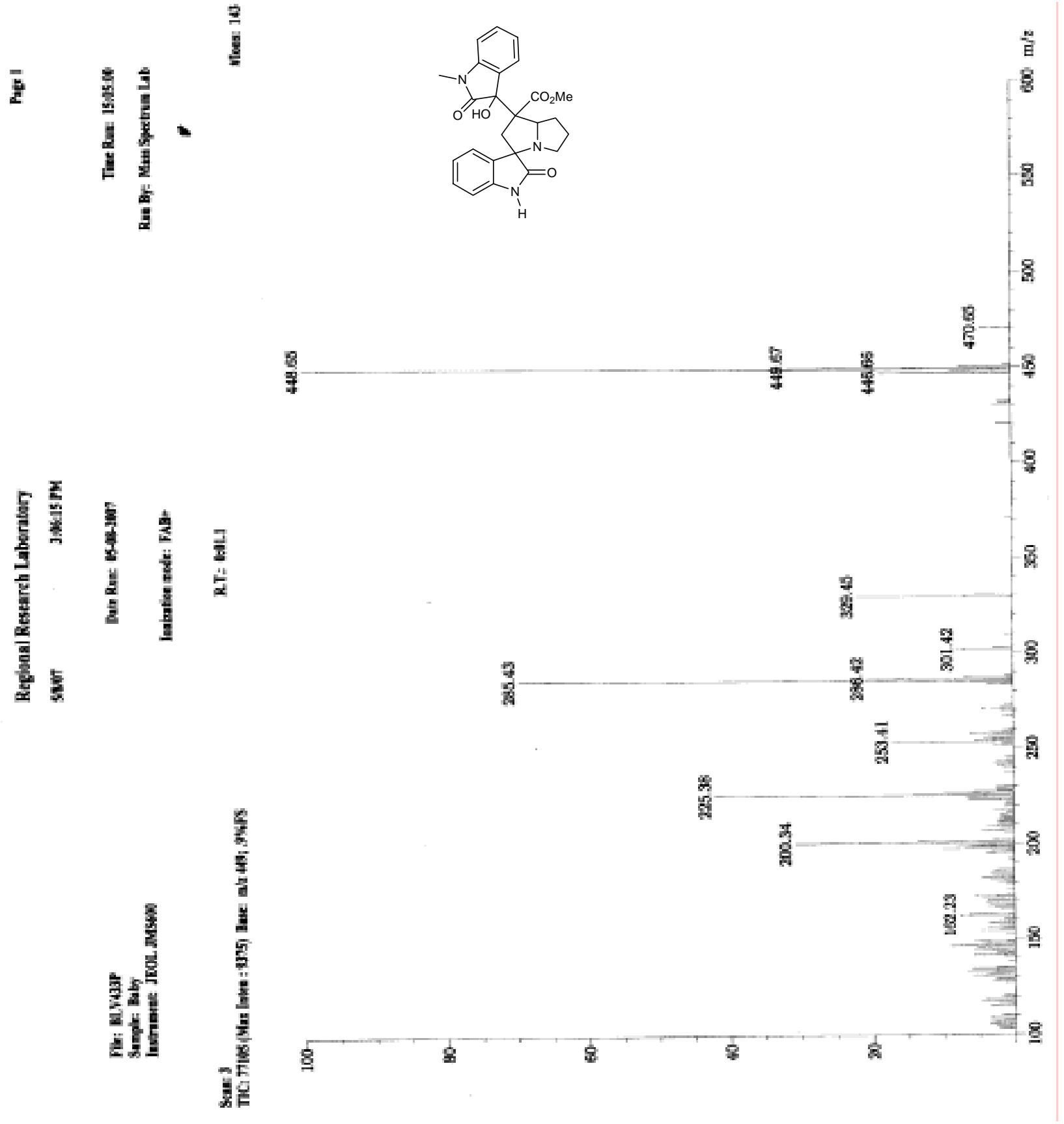

${ }^{1}$ H NMR spectra of 9 


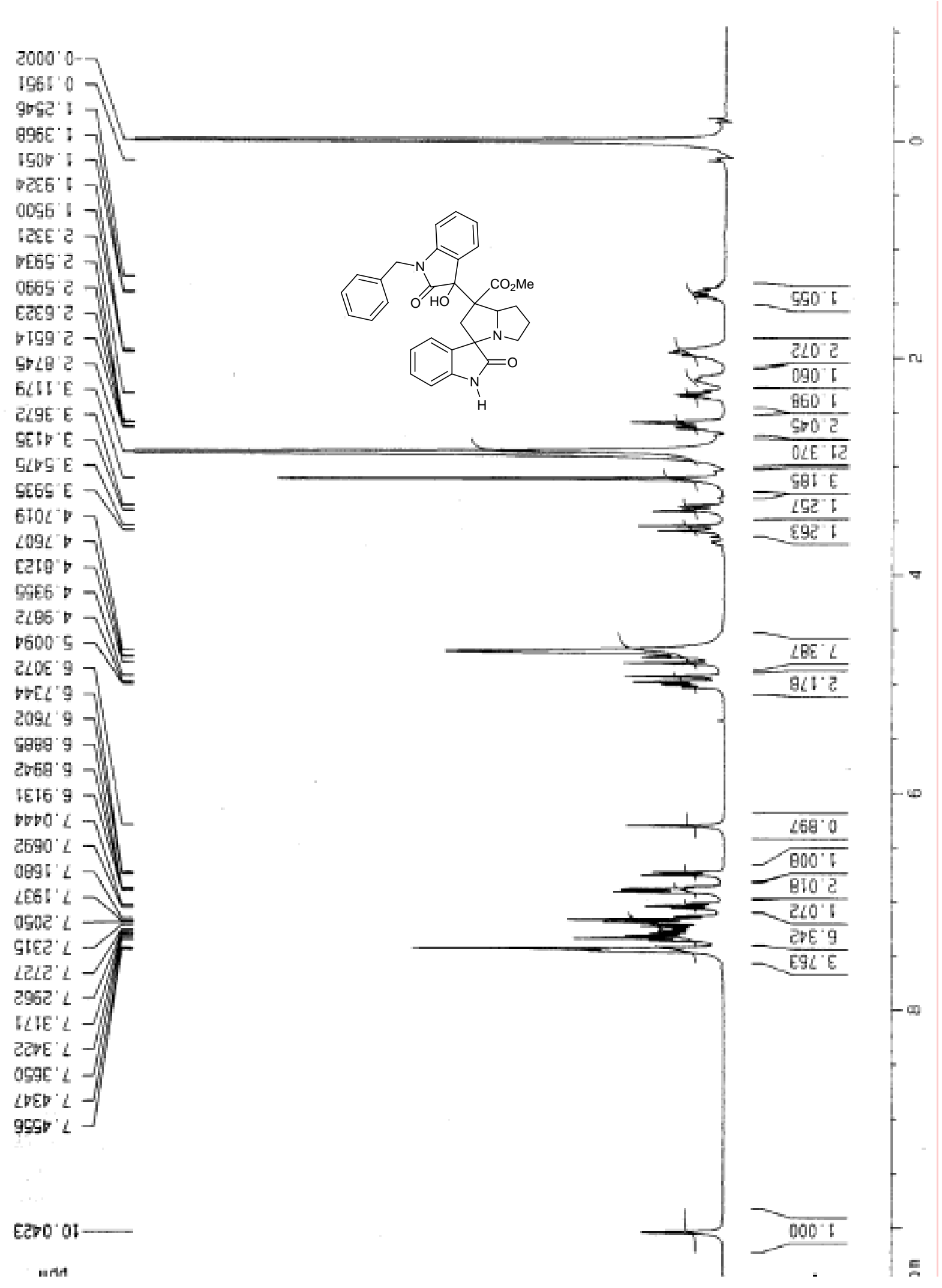

${ }^{13} \mathrm{C}$ 

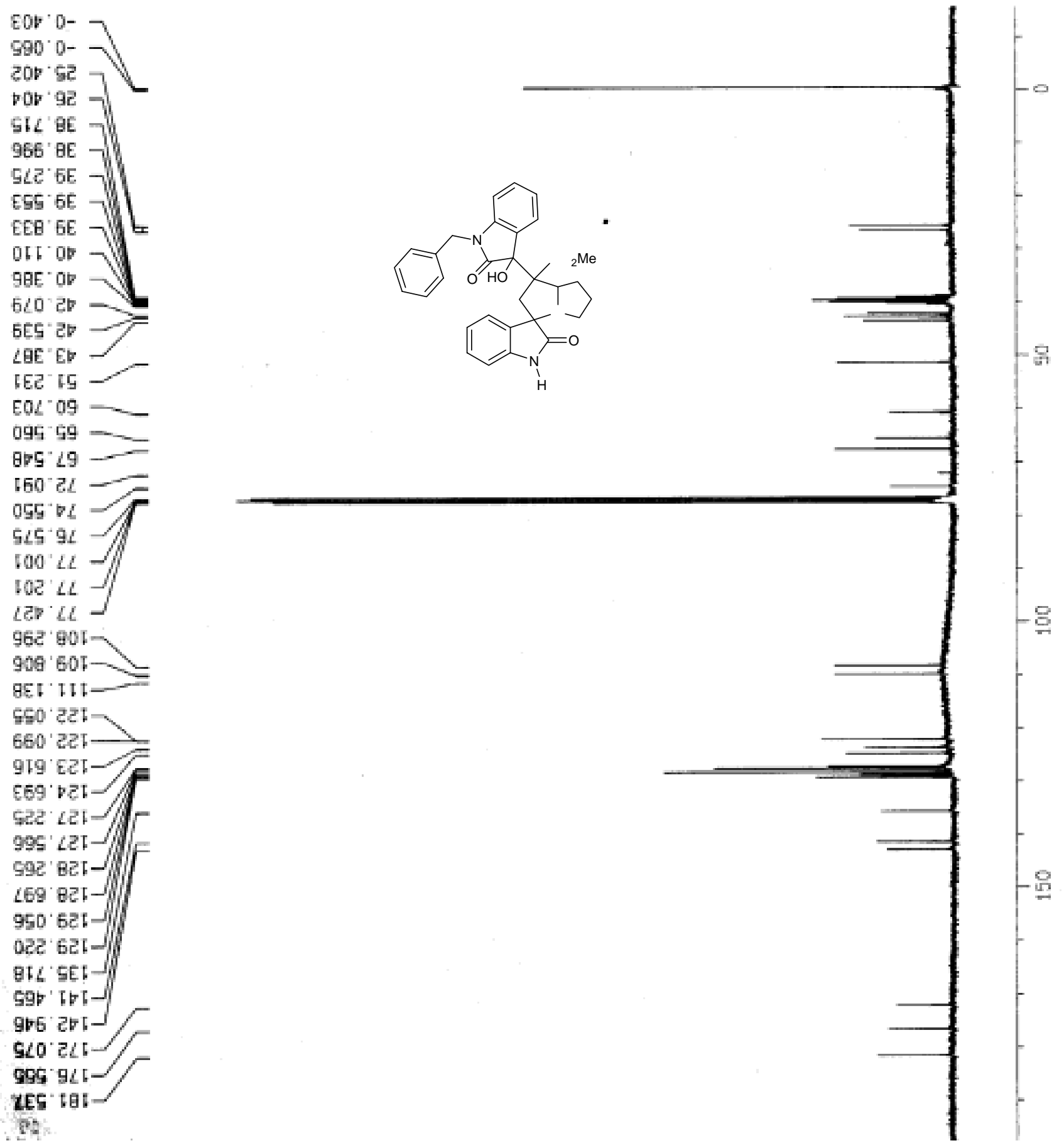

${ }^{1} \mathrm{H}$ NMR spectra of 10 


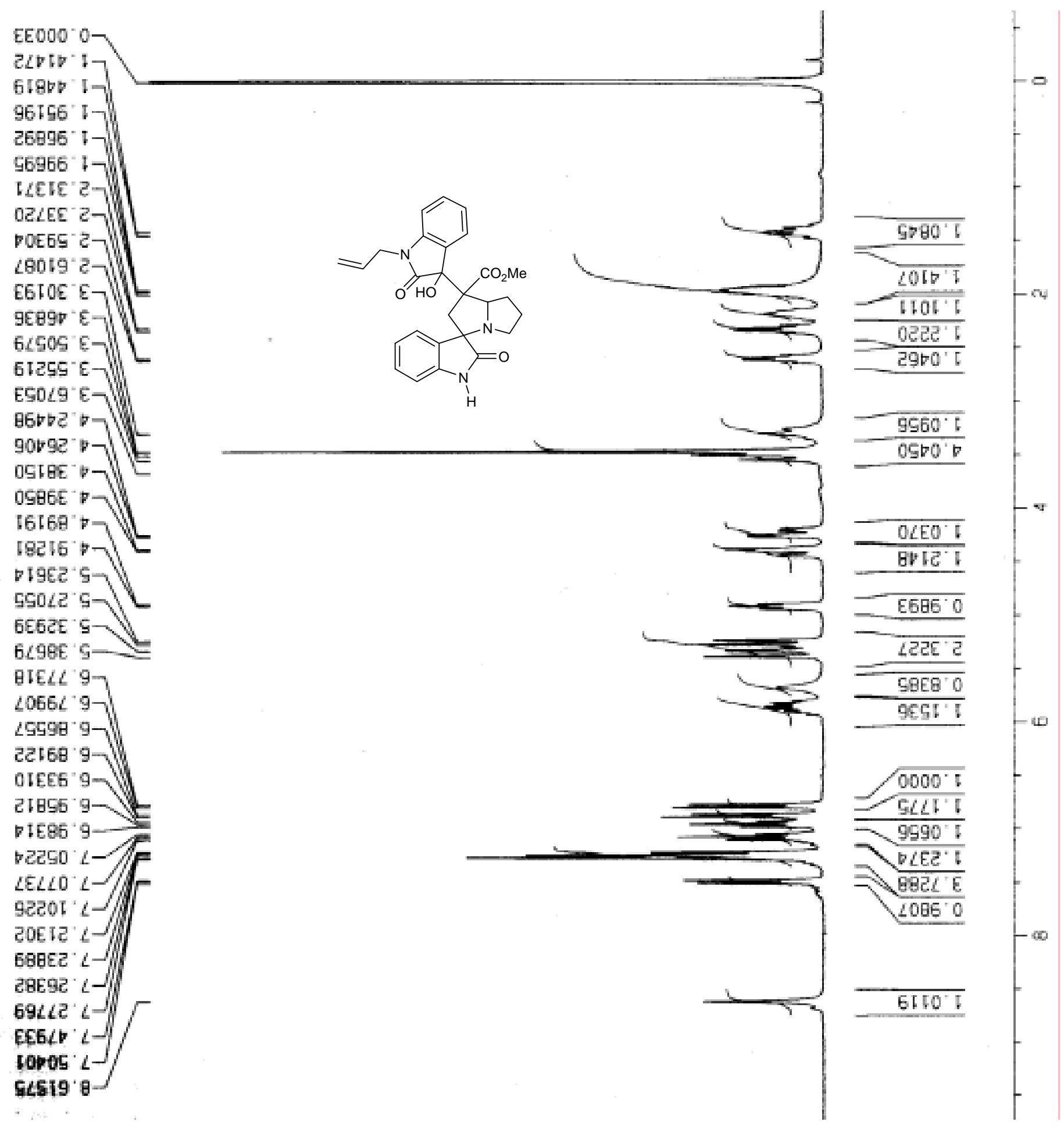


800'0-

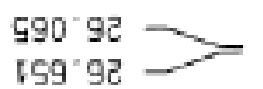

680.

6Е6' ' 55

SBL' $59-$

$\checkmark \angle I \quad 69$

$92 V^{\prime} \mathrm{SL}$

$8 \angle 59 L$

$100^{\circ} \angle C$

b2b' $L L$

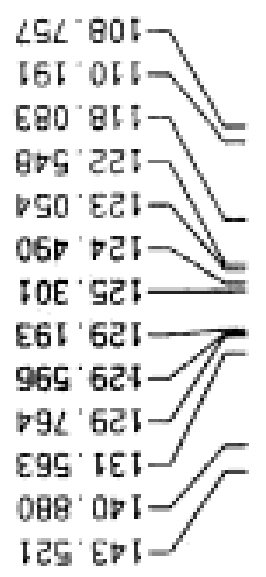

SSV' $2 L 1-$

$68 V^{\circ} 9 \angle \mathrm{F}-$

$\angle 80^{\circ} \mathrm{C} \theta \mathrm{V}$
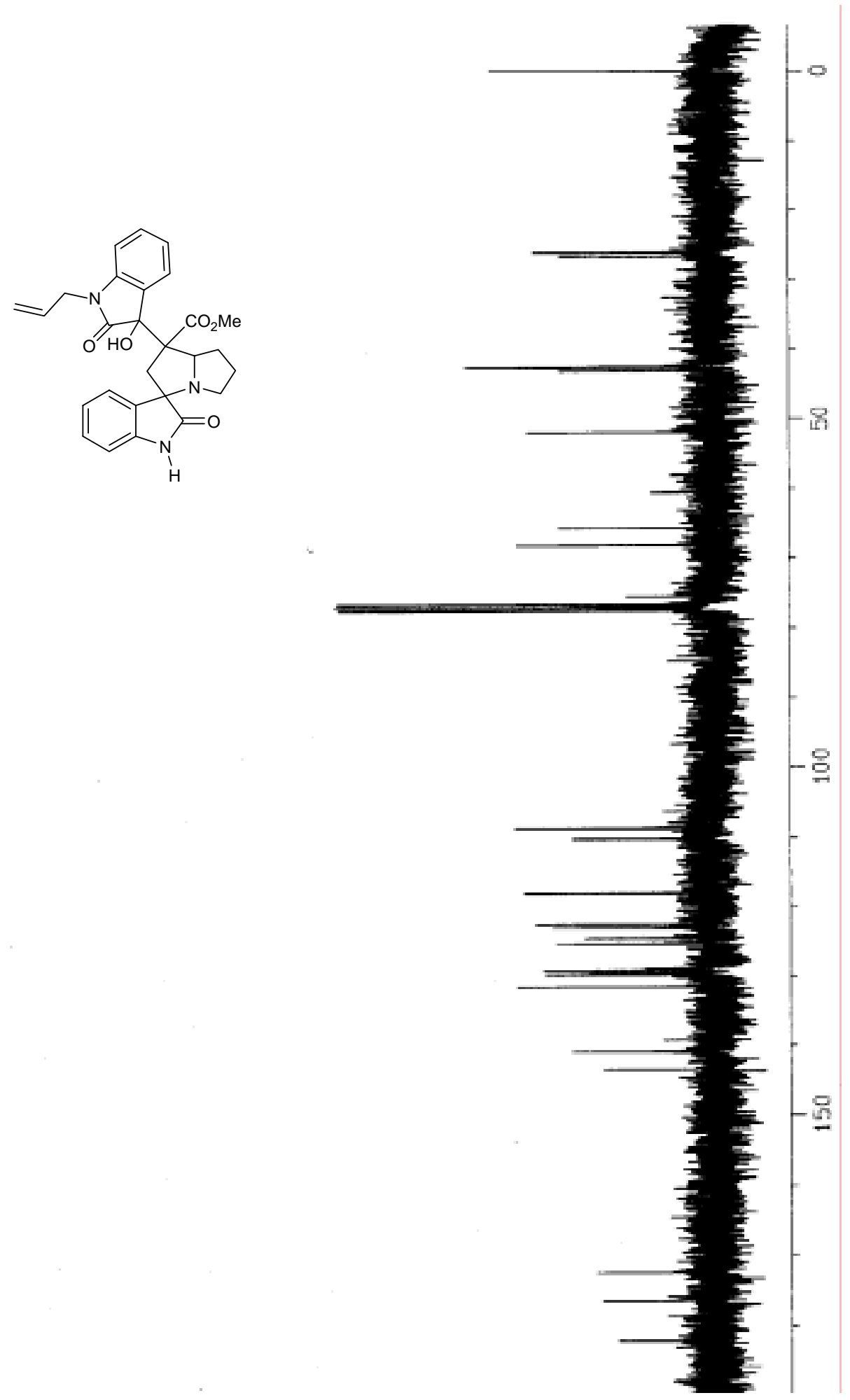

${ }^{1} \mathrm{H}$ NMR spectra of 11 

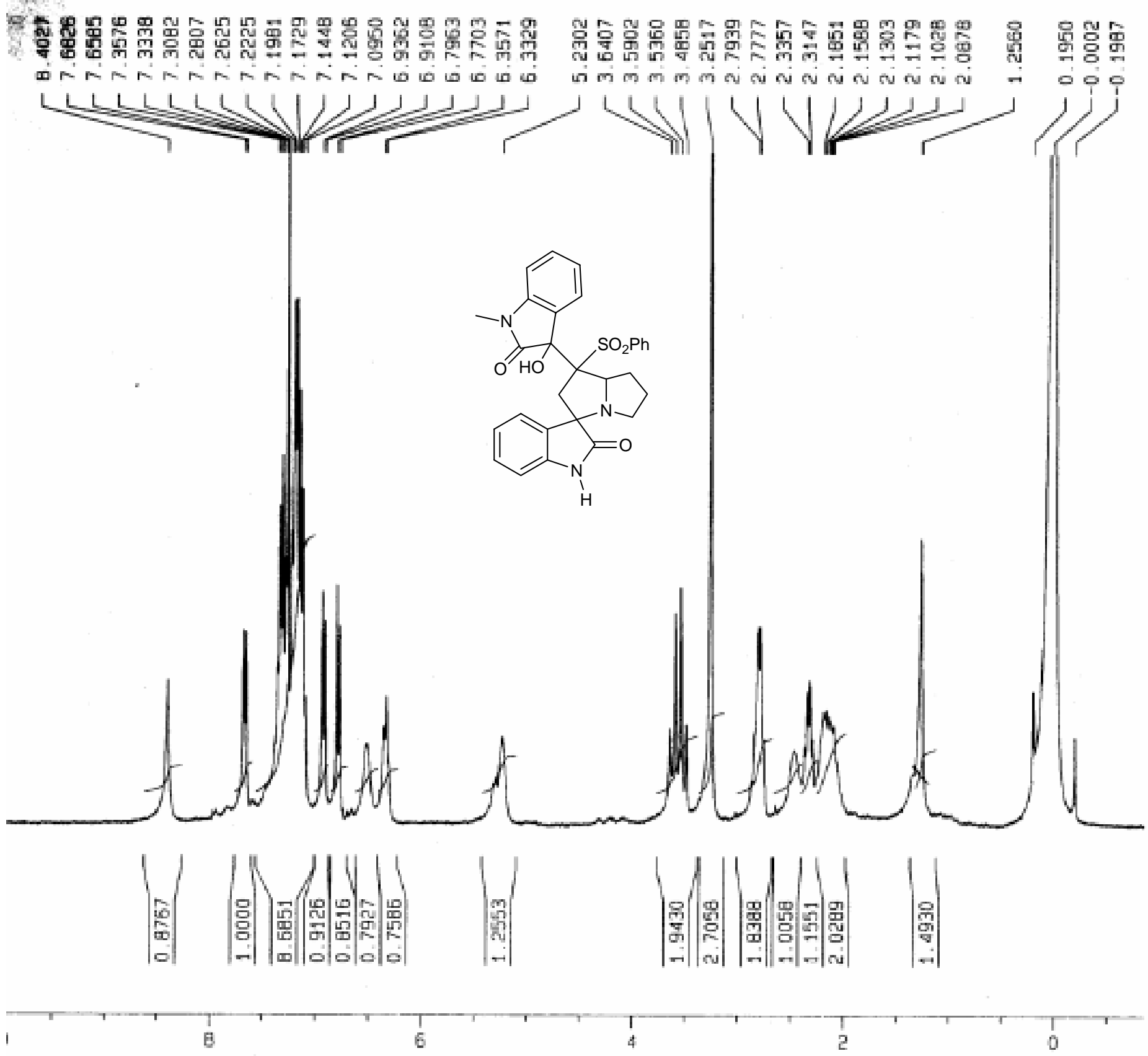


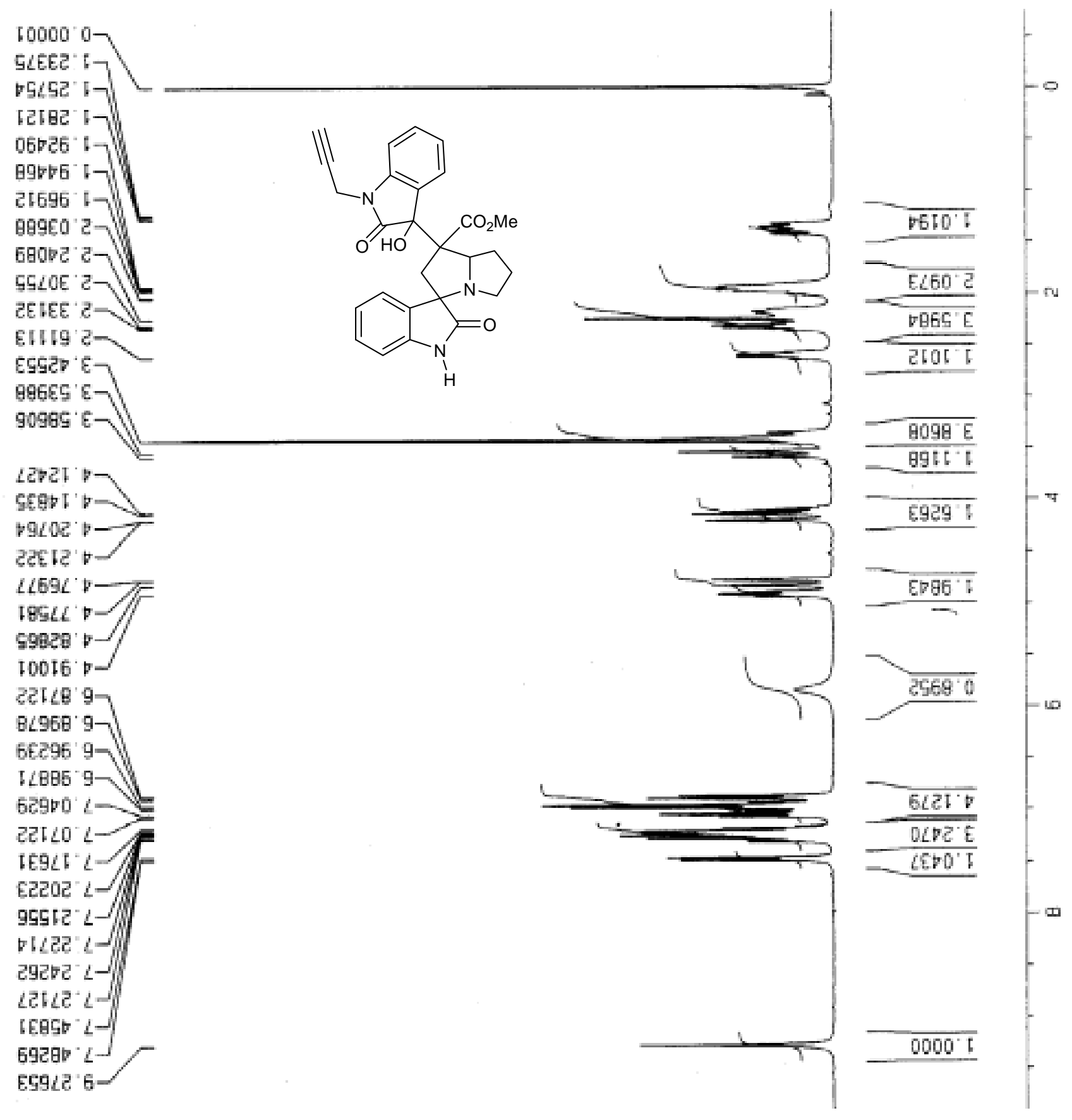


${ }^{13} \mathrm{C}$ NMR spectra of 12

$790^{\prime} 0$

$666^{\prime} \mathrm{gz}$

826.92

$00^{\circ} 62$

$0 \ell 9^{\circ} \mathrm{Eb}$

$950: 2 \mathrm{~V}$

$900.25 \times$

$096^{\circ} 09$

$566 \cdot 59$

SOt'99

9EV'ZL

$1 \angle T \cdot S \angle$

b $\angle G^{\circ} 9 L$

$\angle 66)^{\prime} 9 L$

$2 z b^{\prime} \angle L$

GBV $U L$

DEI 96

268'90'

टदb 0 เ

$\angle B^{\circ}$ 'C2 5

890 'є己!-

6DE' bटt

$8 \angle C^{\prime} \mathrm{G} L 5-$

$880.625-7$

$2 / 2 \cdot 6 \mathrm{ct}-/$

E6E 625-/

$\varepsilon 9 L$ '62t-

$550^{\circ} \mathrm{WT}-$

IEE'zण
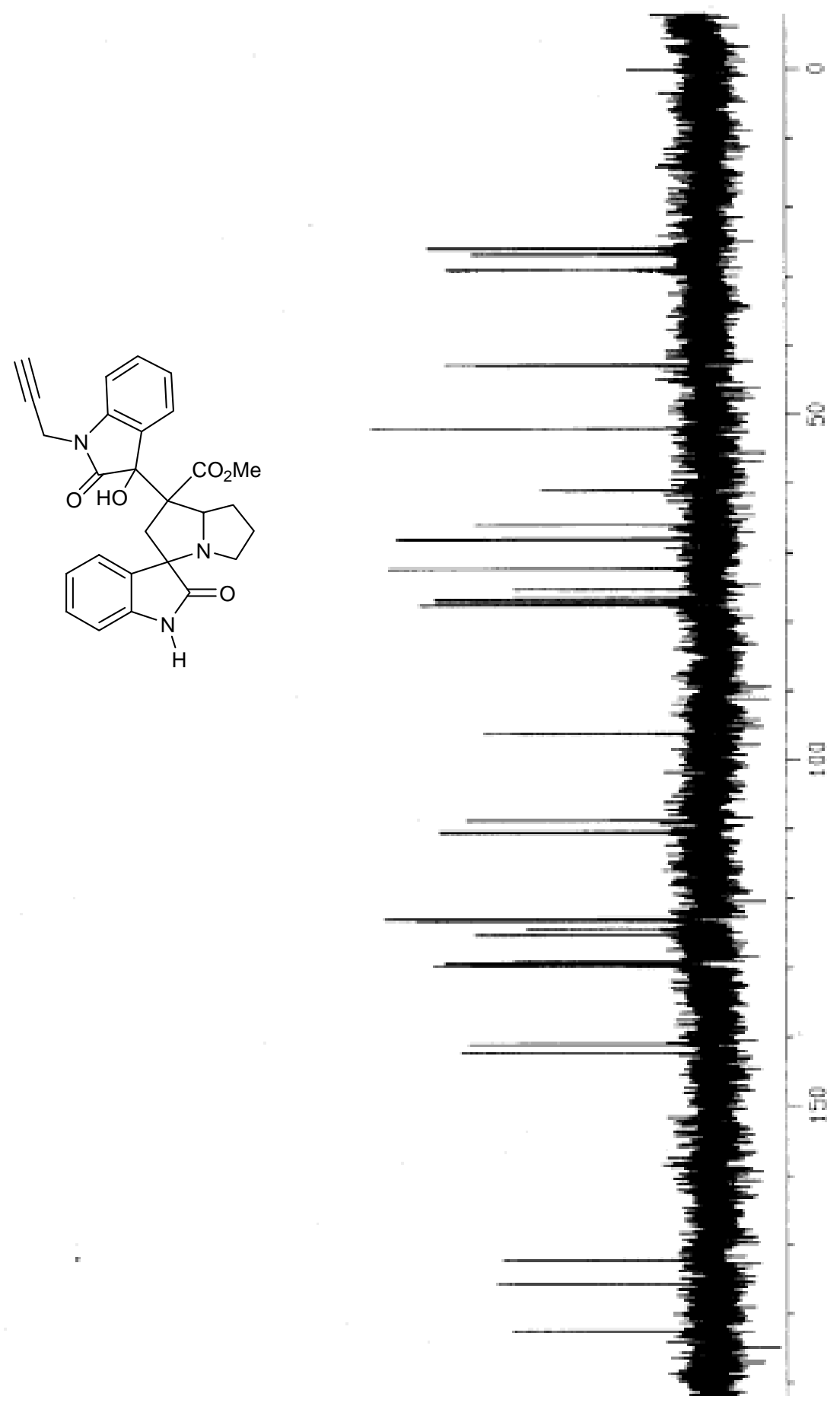

Eg: $221-$

0 se st - -

IE " 281 


\section{${ }^{1} \mathrm{H}$ NMR spectra of 13}

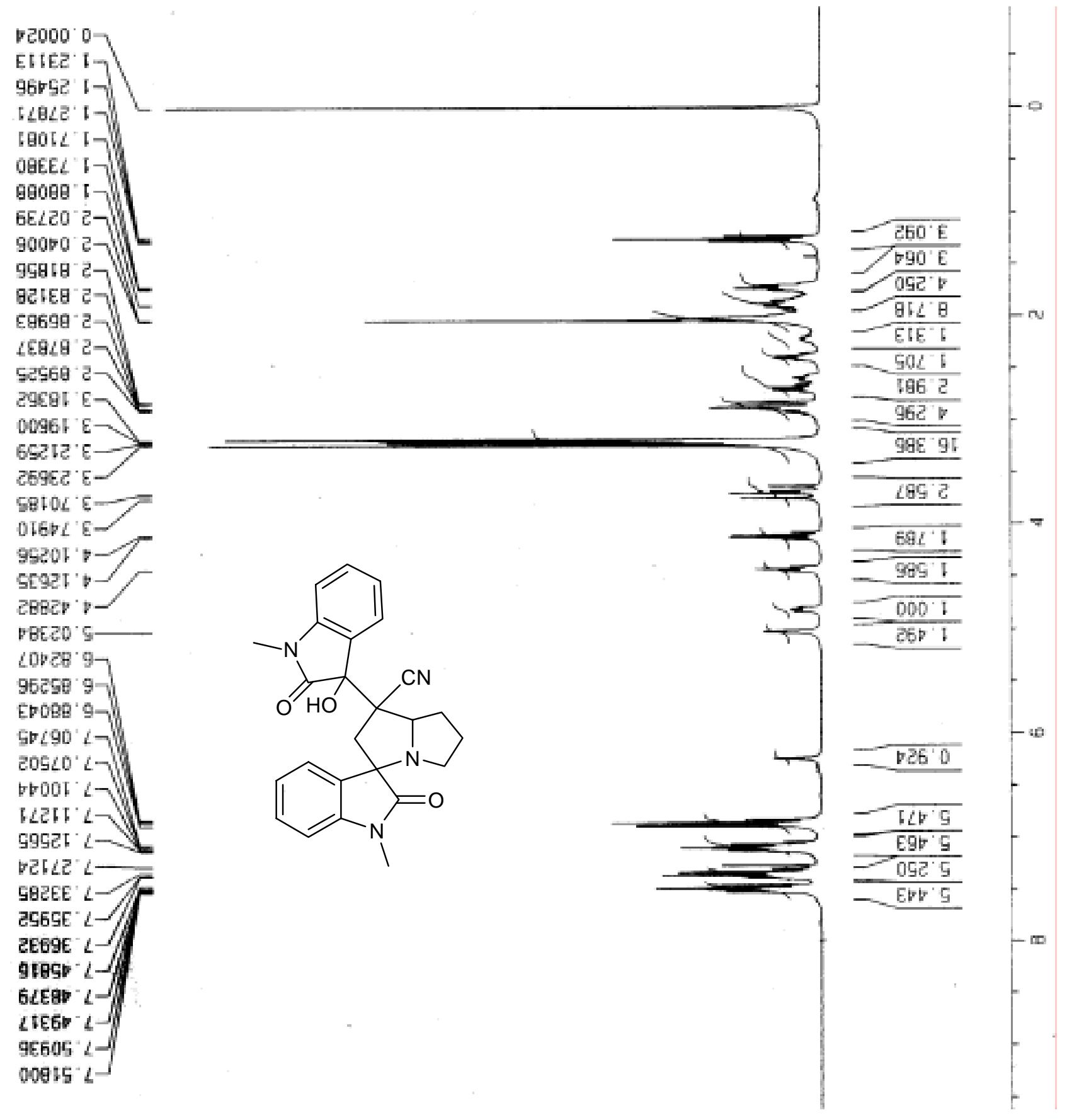


FAB mass of 13

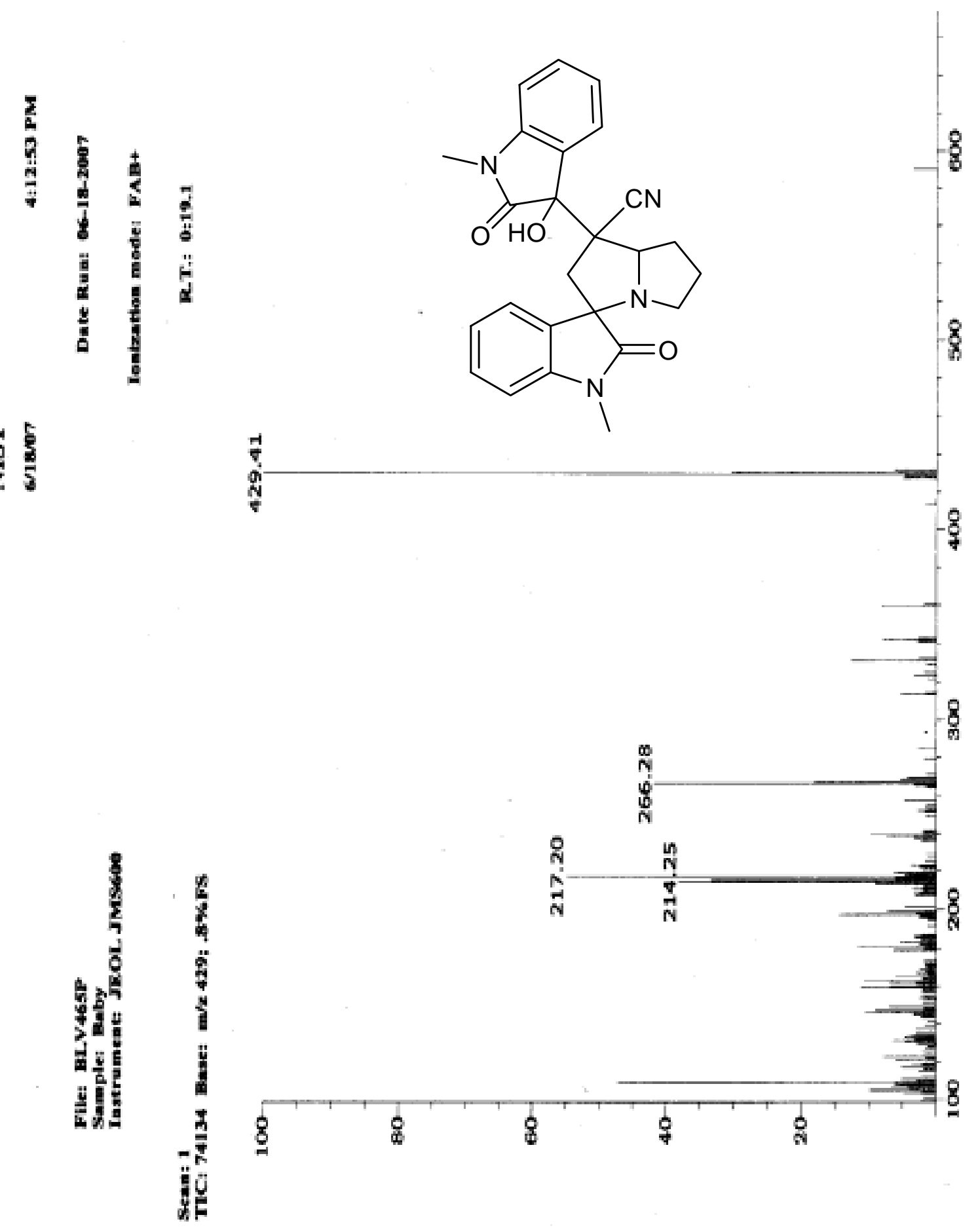




\section{${ }^{1}$ H NMR spectra of 15}

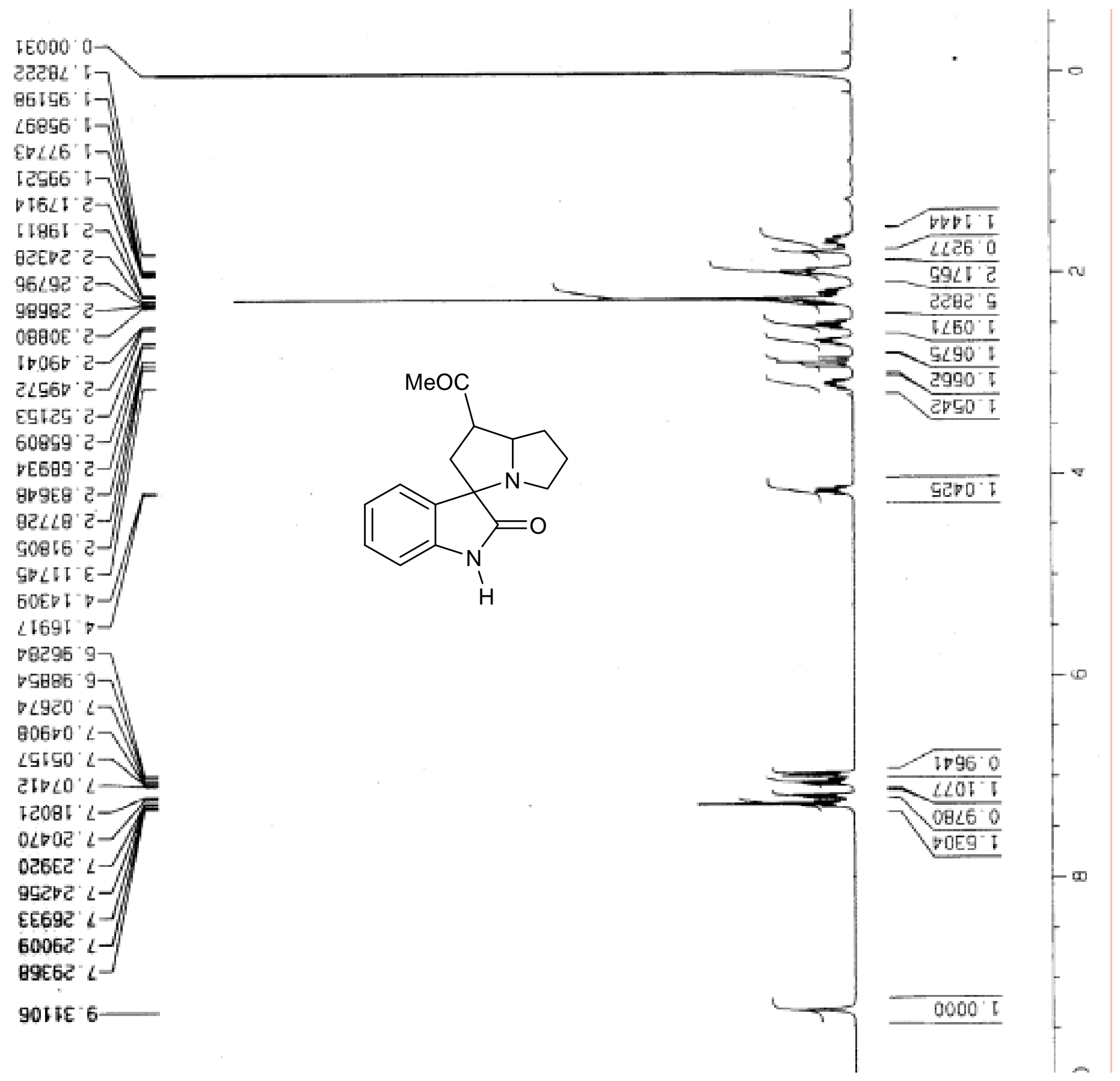




\section{FAB mass of 15}
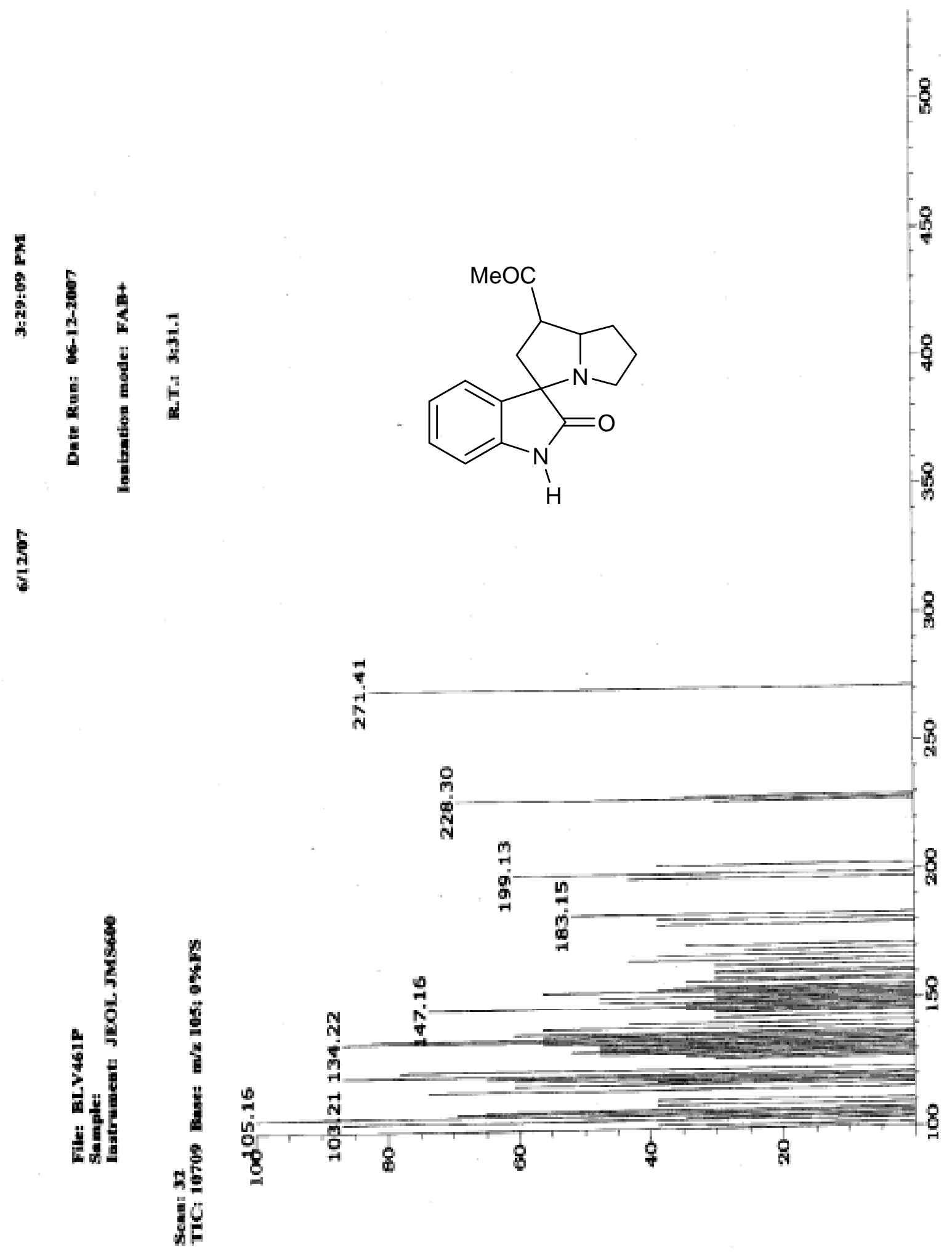


\section{${ }^{1} \mathrm{H}$ NMR spectra of 18}

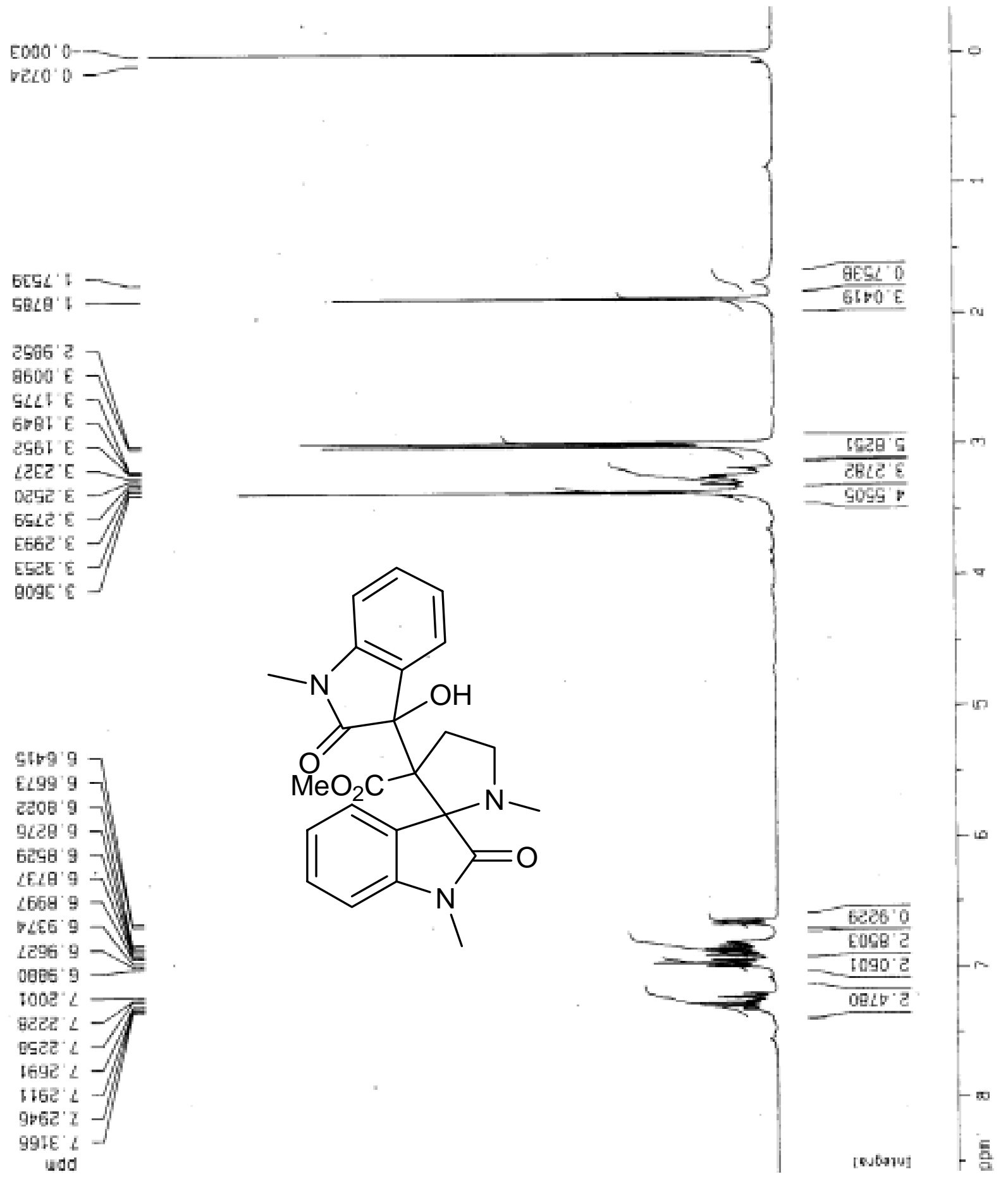




\section{${ }^{13} \mathrm{C}$ NMR spectra of 18}

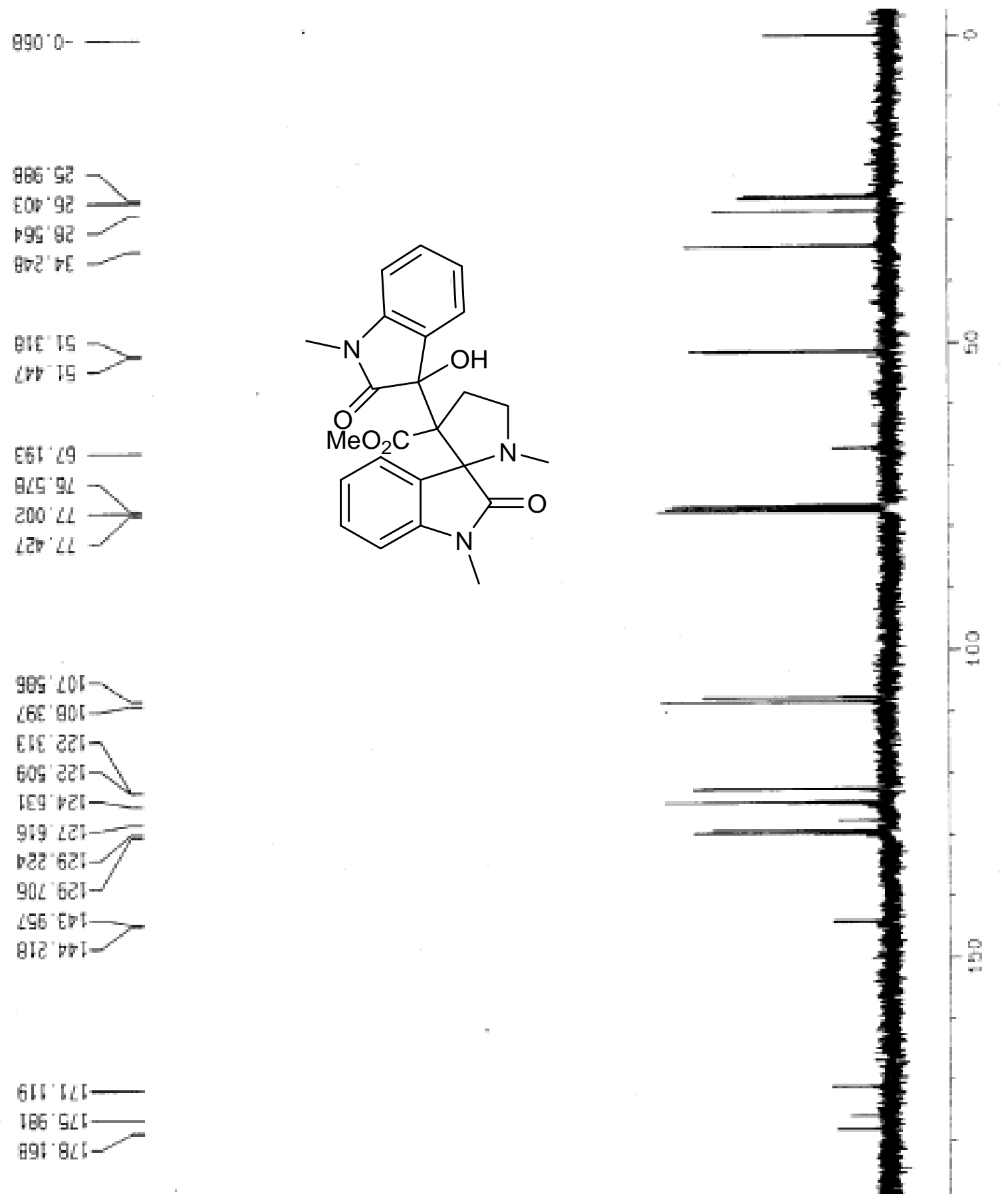




\section{${ }^{1}$ H NMR spectra of 19}

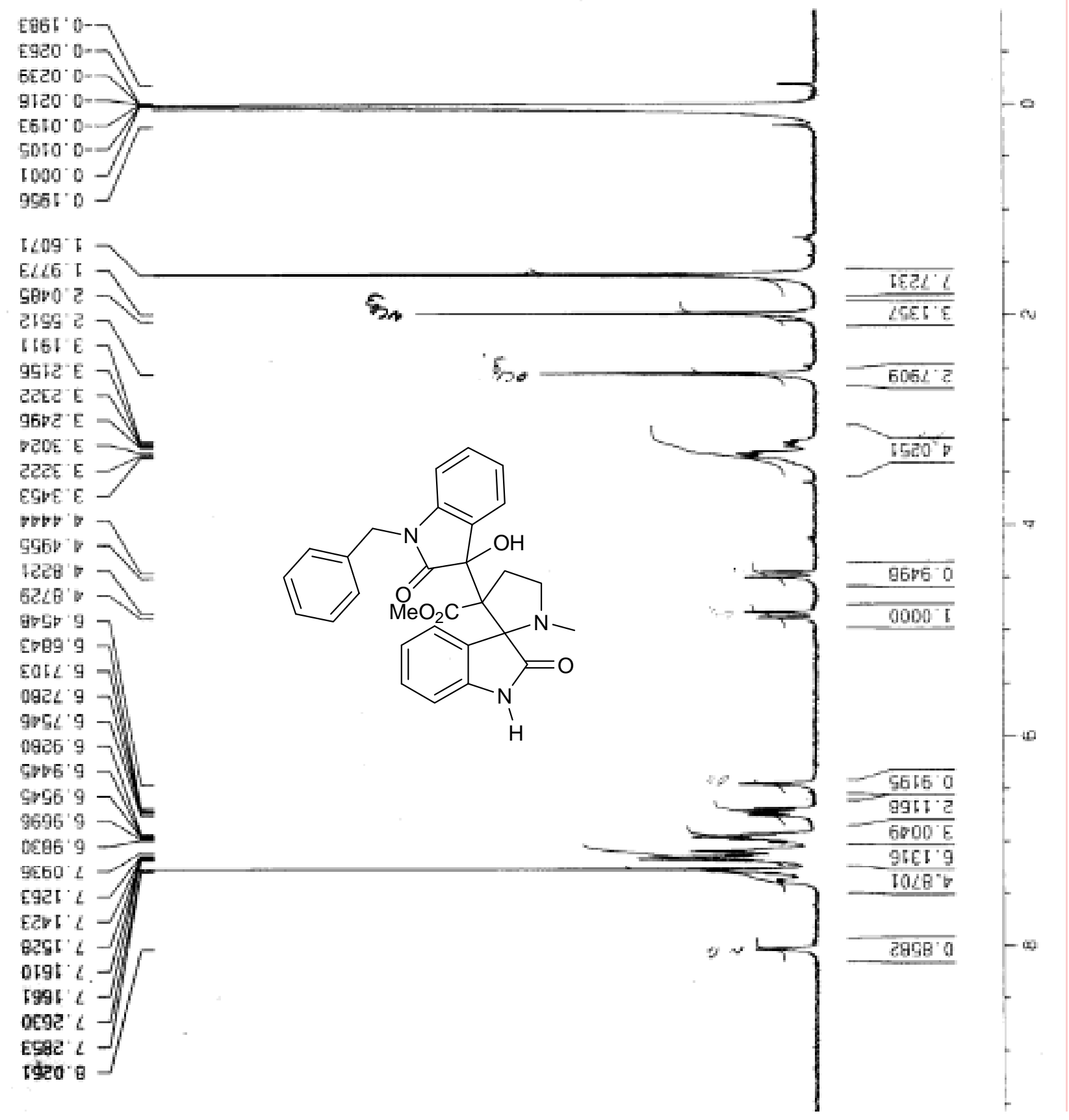


${ }^{1}$ H NMR spectra of 20

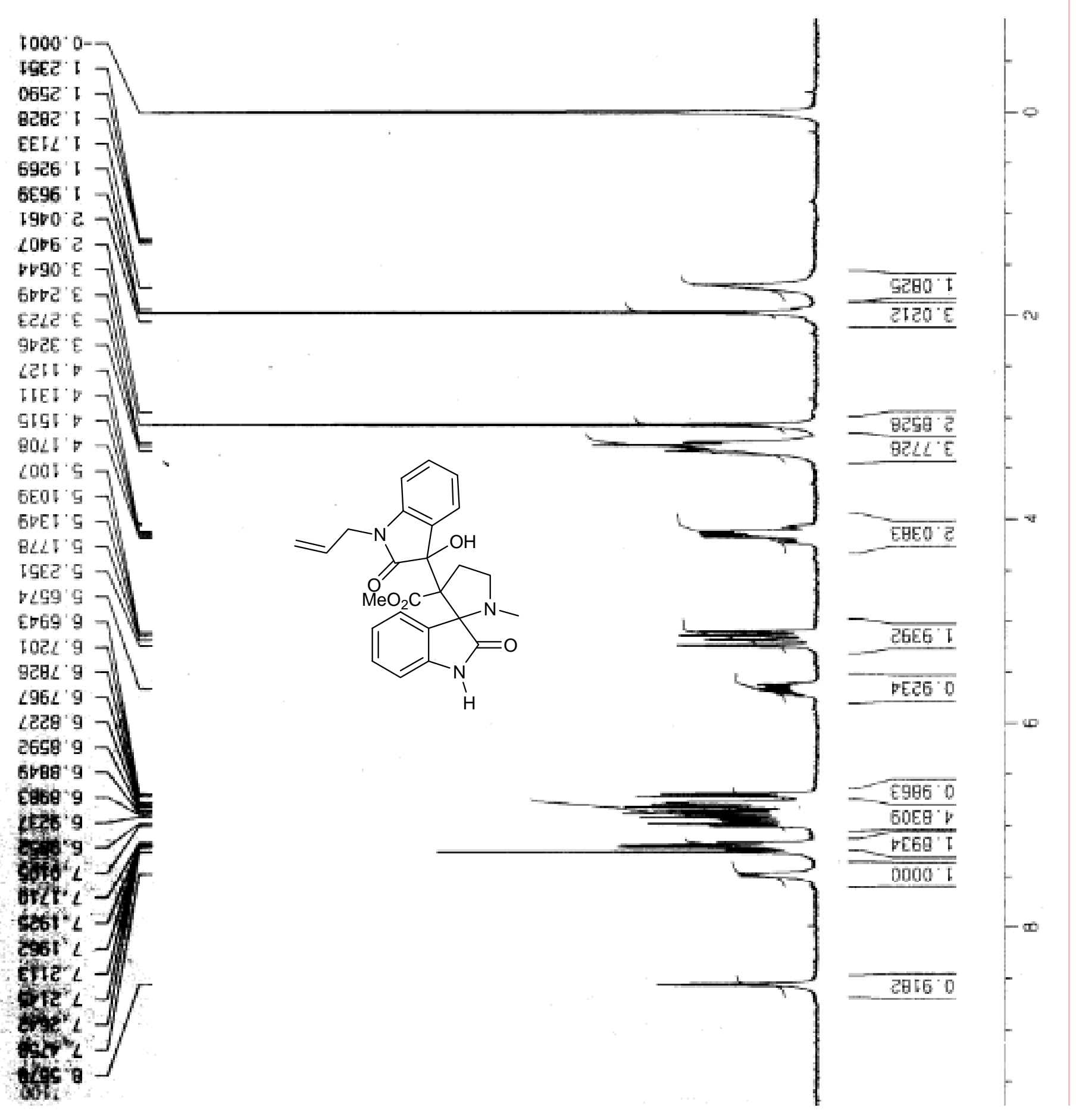


${ }^{13} \mathrm{C}$ NMR spectra of 20

$680^{\prime} 92$

QIE ' $\square$

$80 \varepsilon^{\prime} \mathrm{ct}$

$\angle 90^{\circ}$ IS $>$

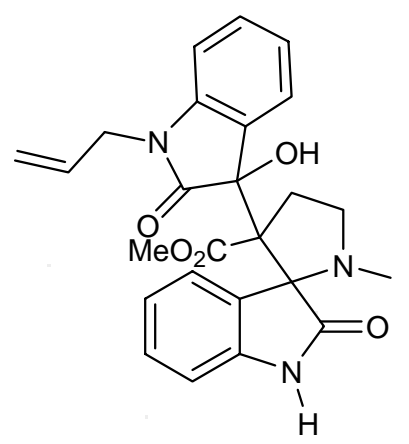

$616 \cdot 99$

$9 \angle S^{\circ} 9 L$

$000^{\circ} \angle L$

$\checkmark 20<L$

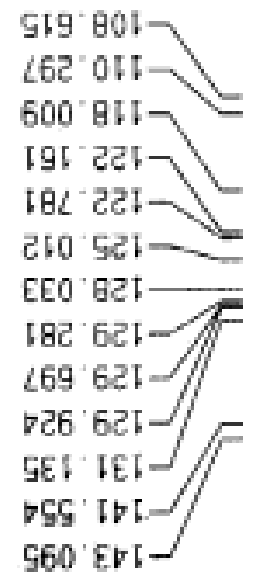

$\angle D D^{\prime} L \angle D-$

It ' $9 \angle \mathrm{C}$

er8 $621-$

to.

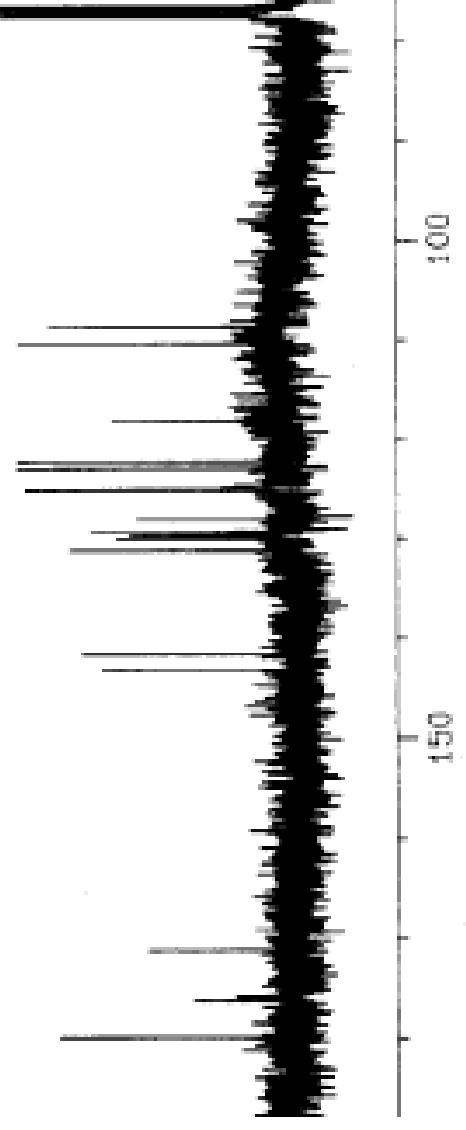




\section{${ }^{1} \mathrm{H}$ NMR spectra of 21}

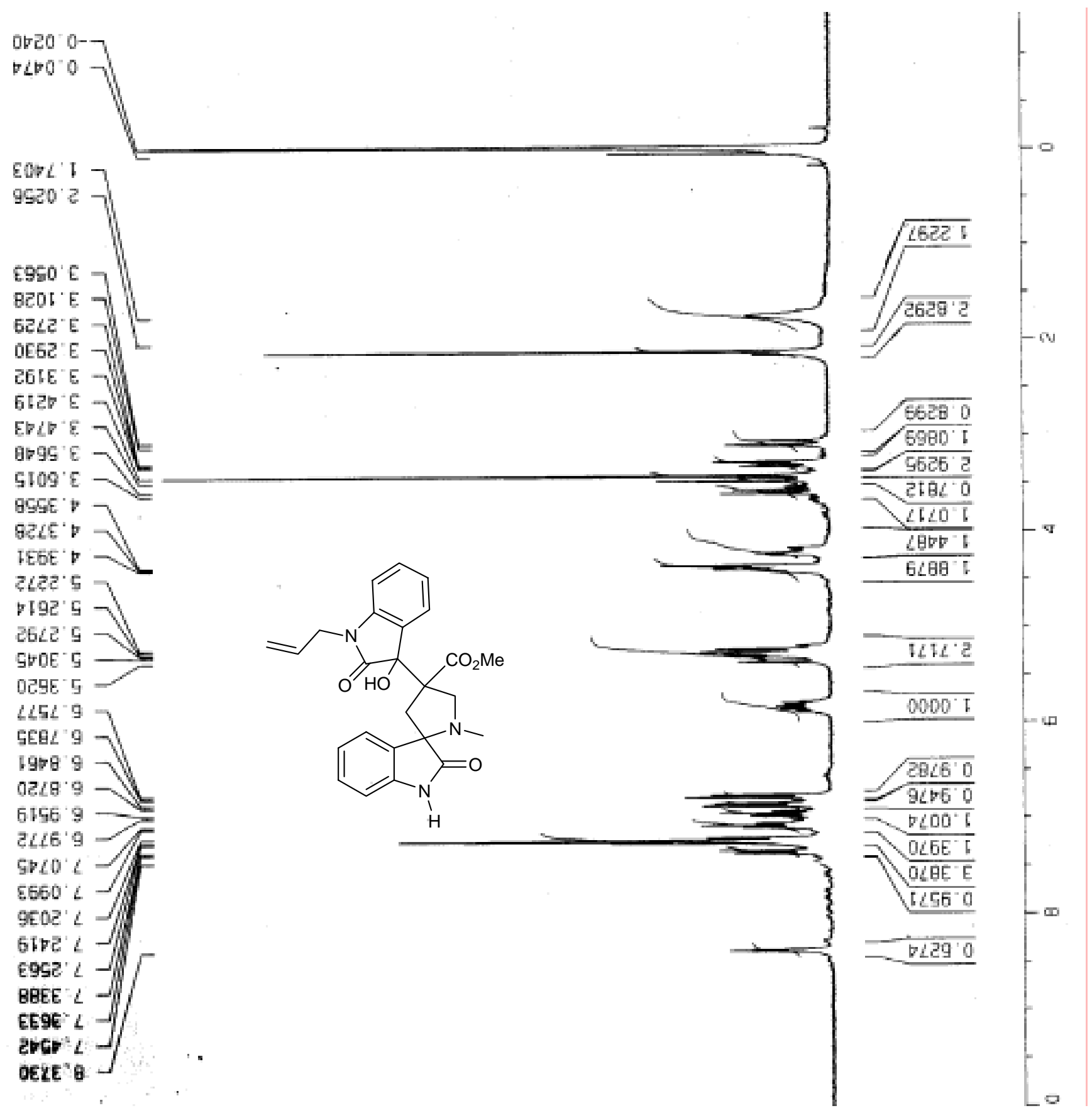


${ }^{13} \mathrm{C}$ NMR spectra of 21
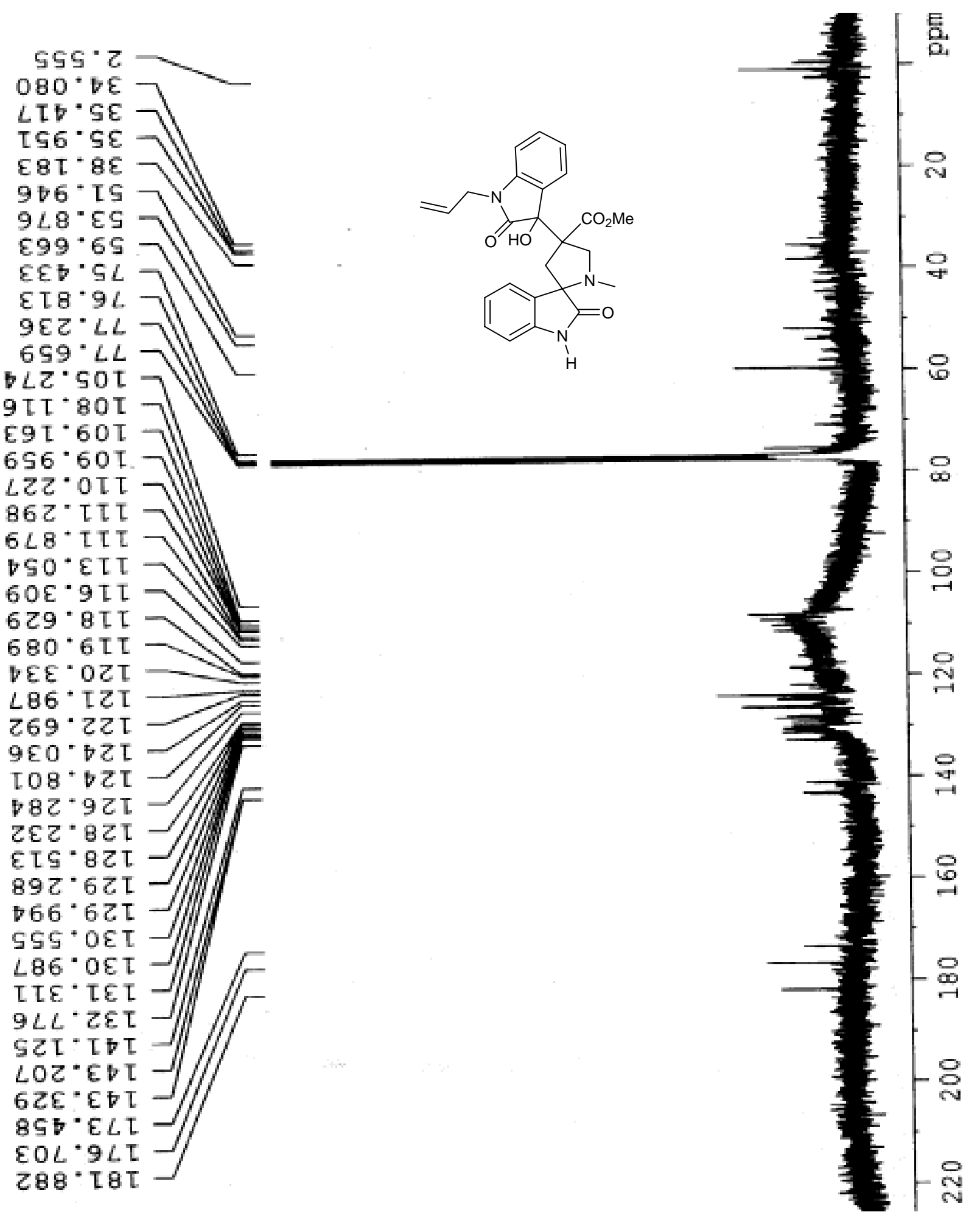
${ }^{1} \mathrm{H}$ NMR spectra of 22

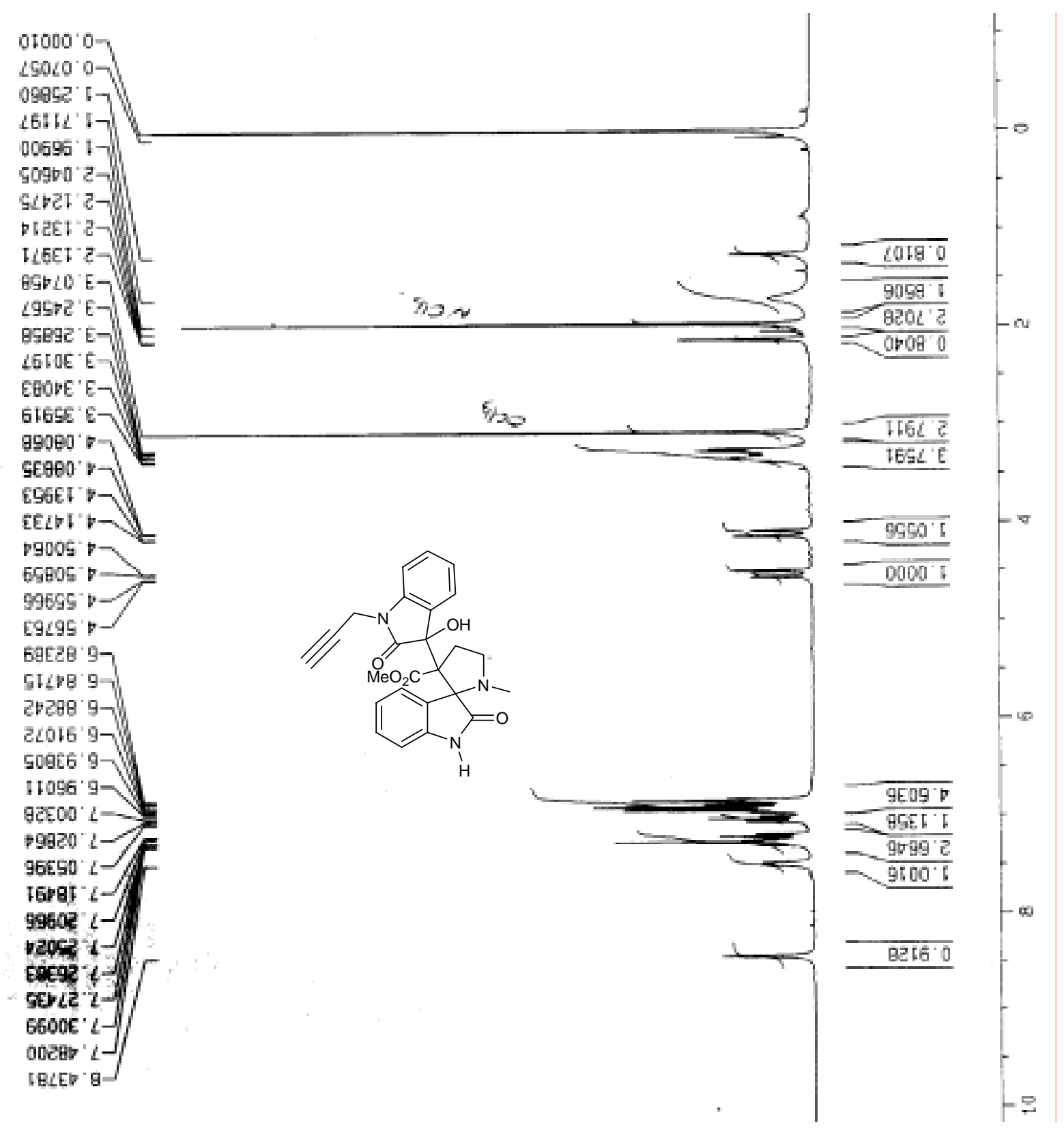




\section{${ }^{1} \mathrm{H}$ NMR spectra of 23}

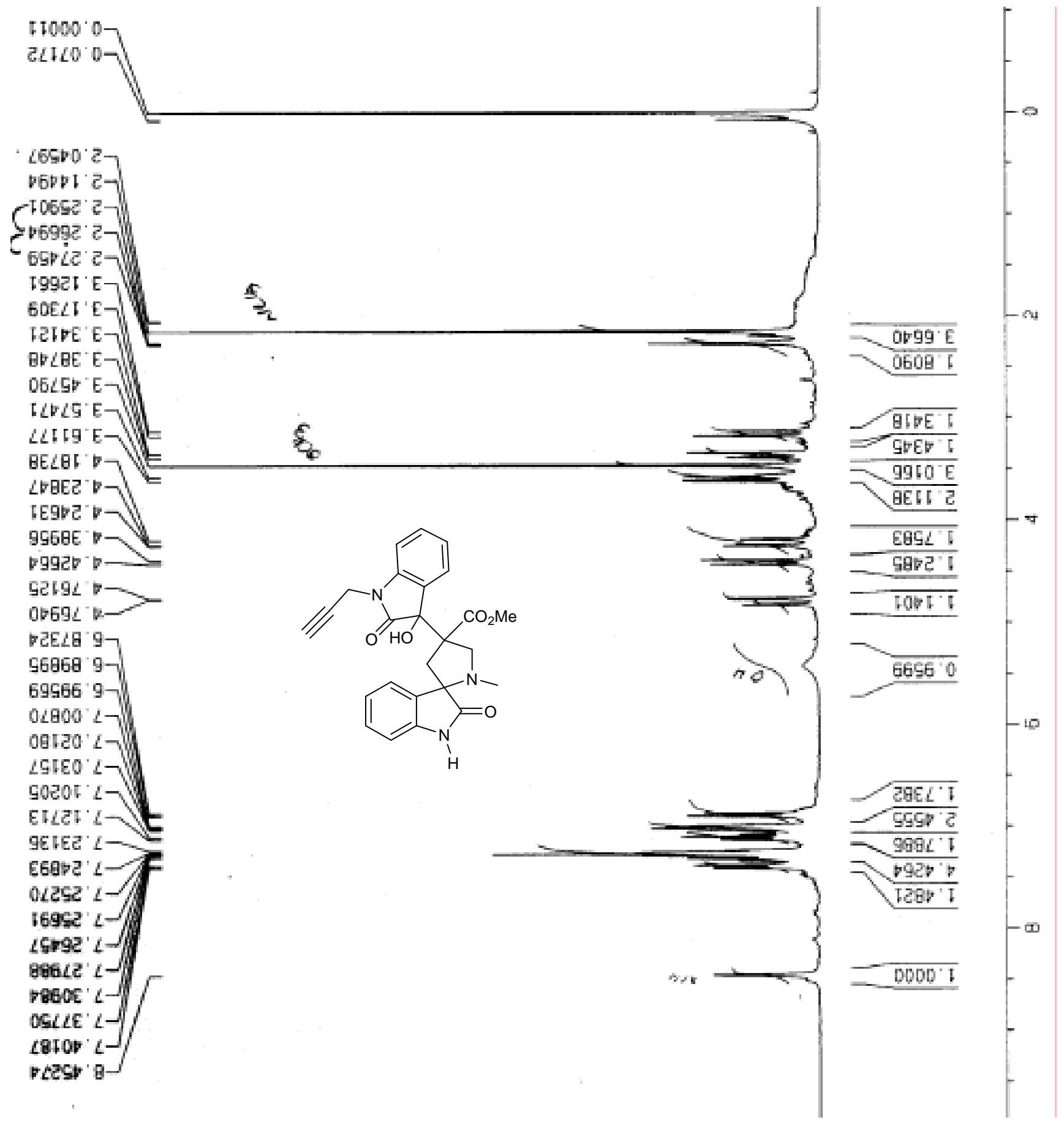


${ }^{1} \mathrm{H}$ NMR spectra of 24

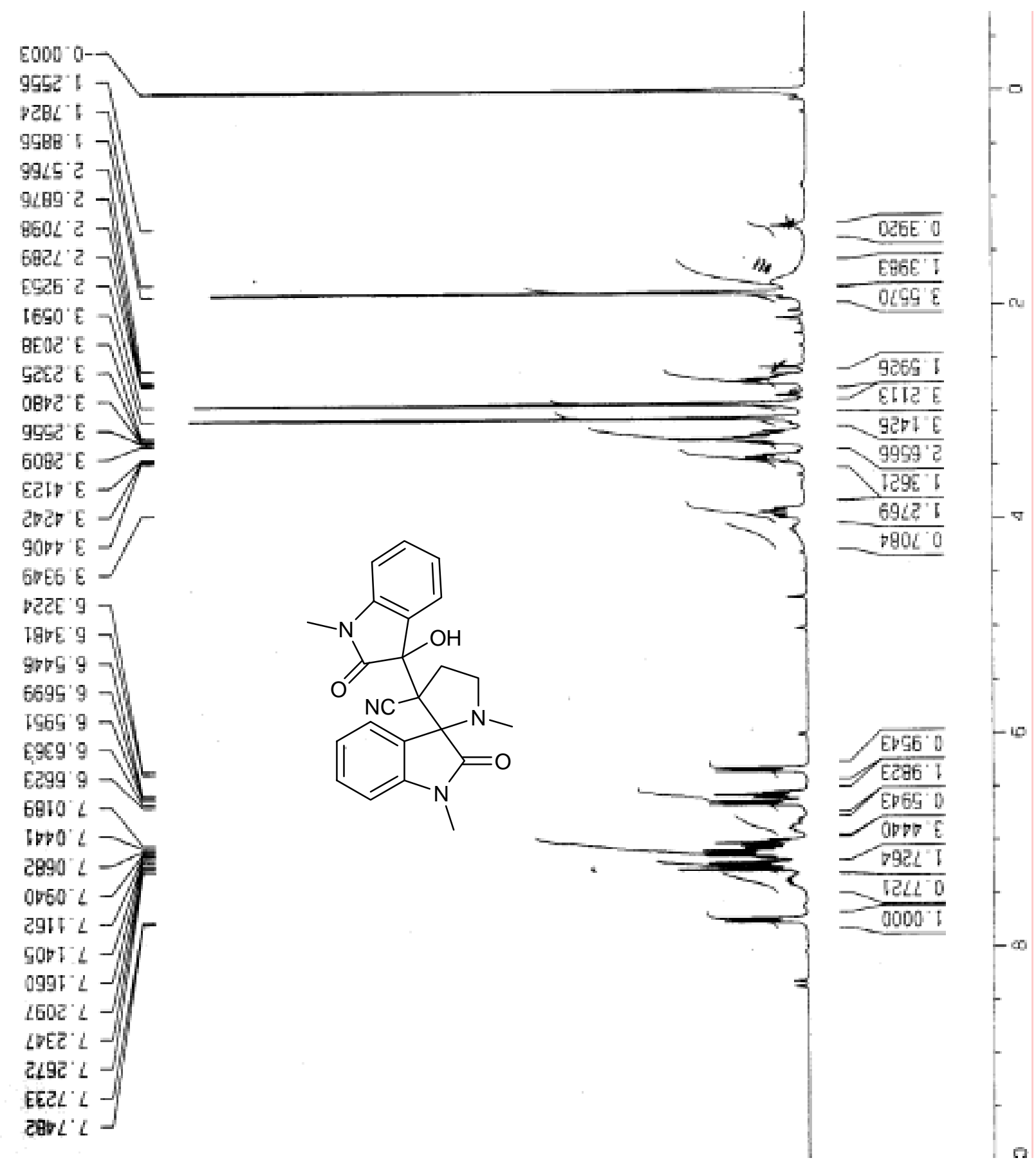




\section{${ }^{1} \mathrm{H}$ NMR spectra of 25}

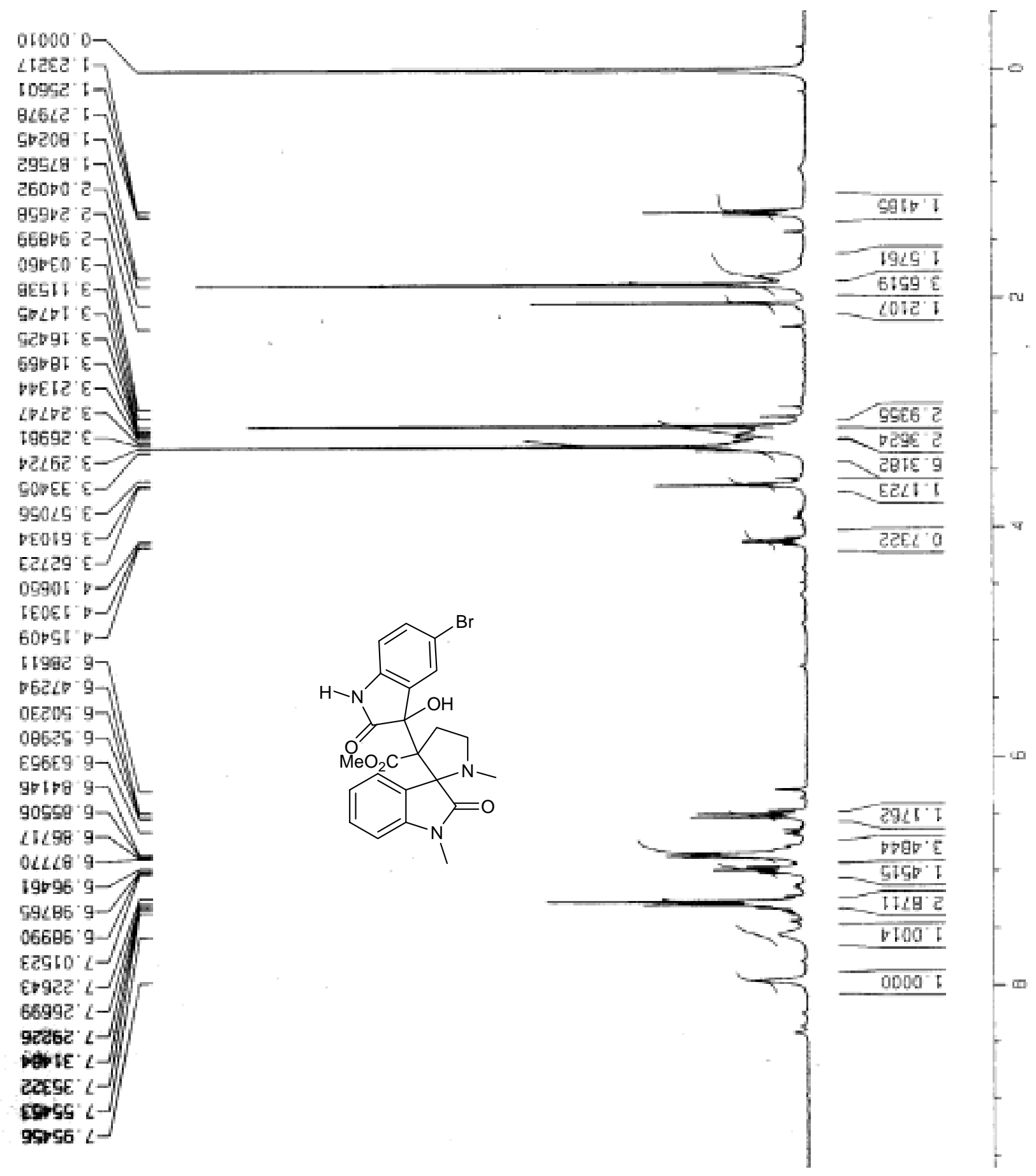


${ }^{13} \mathrm{C}$ NMR spectra of 25

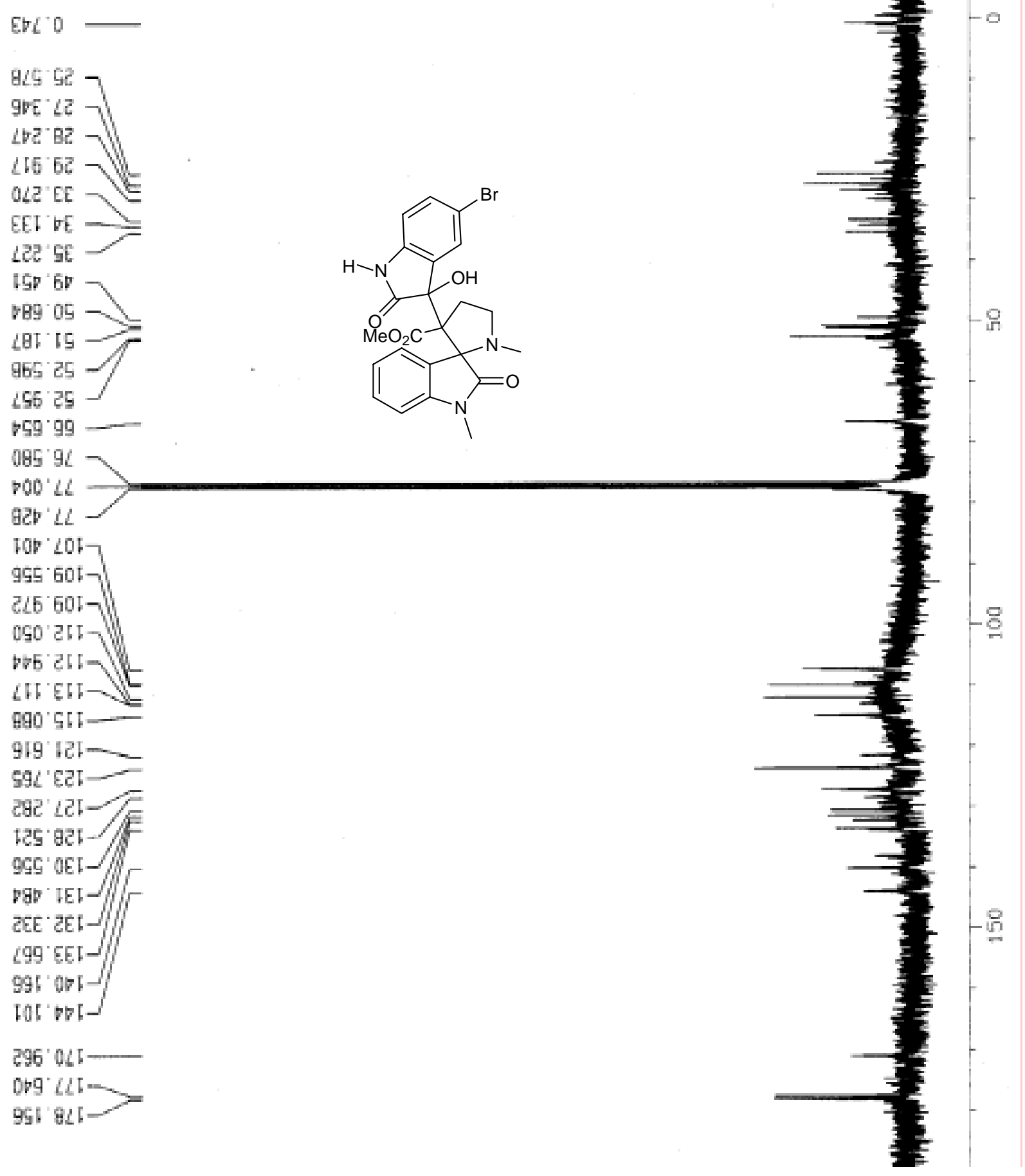




\section{${ }^{1} \mathrm{H}$ NMR spectra of 26}

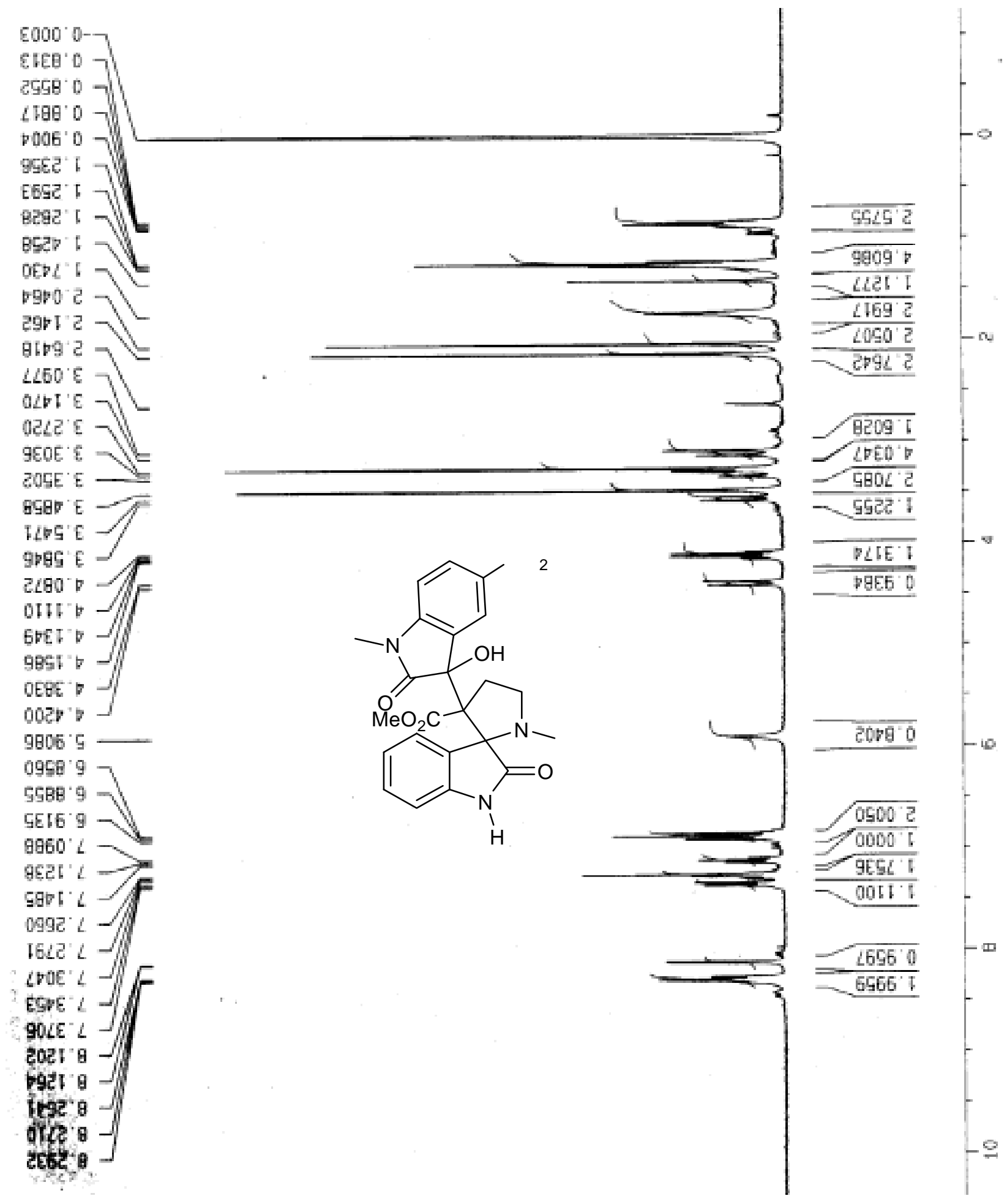


${ }^{13} \mathrm{C}$ NMR spectra of 26

$290^{\circ} 0-$

$D E 0^{\circ} 12-$
$979^{\prime} 92-$

07t'

$8 \angle \theta^{\circ} \angle E-$

E 16.25

O20 $1 \mathrm{ES}$

$289 \div$

IEE' 65

GLL'OL

$616,6 L$

$085^{\circ} 9 l$

$700^{\circ} 16$

$\angle 20 ' L L$

$669^{\circ} \mathrm{COt}-$

625 $501-$

$20 G^{\prime} 60 \mathrm{~T}$

29106

ZLS 02T

$\checkmark L C E C$

E20 Gેઈ

gET $\angle 2 t-$

टहट $6 ट 5-$

$\rightarrow 65.6512$

ब19.6टा

gEL OOH

Oge' $\mathrm{EDT}-$

veg 601<smiles>COC(=O)C1(C2(C)CCN(C)C23C(=O)Nc2ccccc23)C(=O)N(C)c2ccc(C)cc21</smiles>

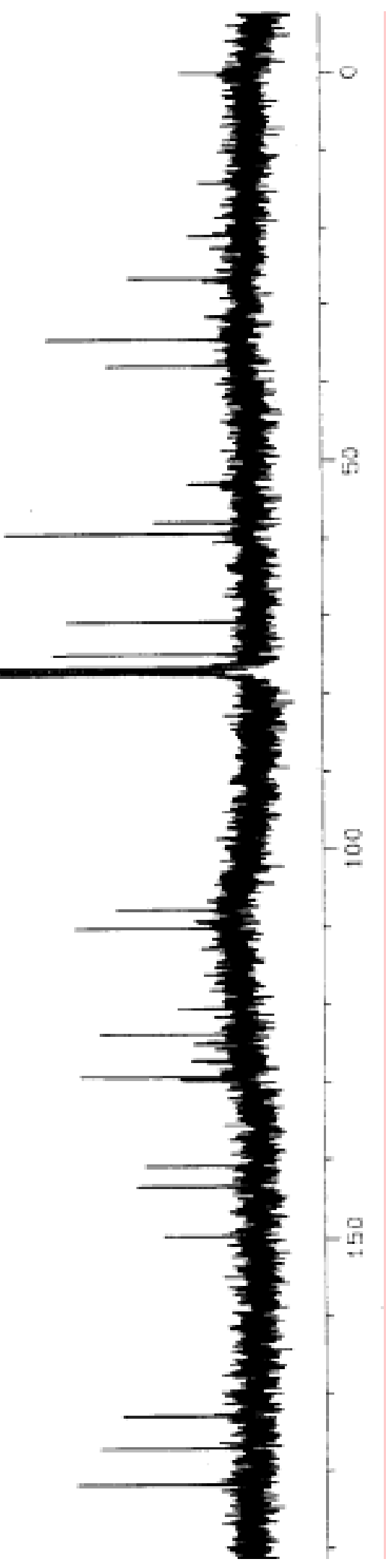

228 ' $2 \angle 1-$

$086.9 \angle 5$

899' เดเ 


\section{${ }^{1} \mathrm{H}$ NMR spectra of $26\left(\mathrm{D}_{2} \mathrm{O}\right.$ exchanged $)$}

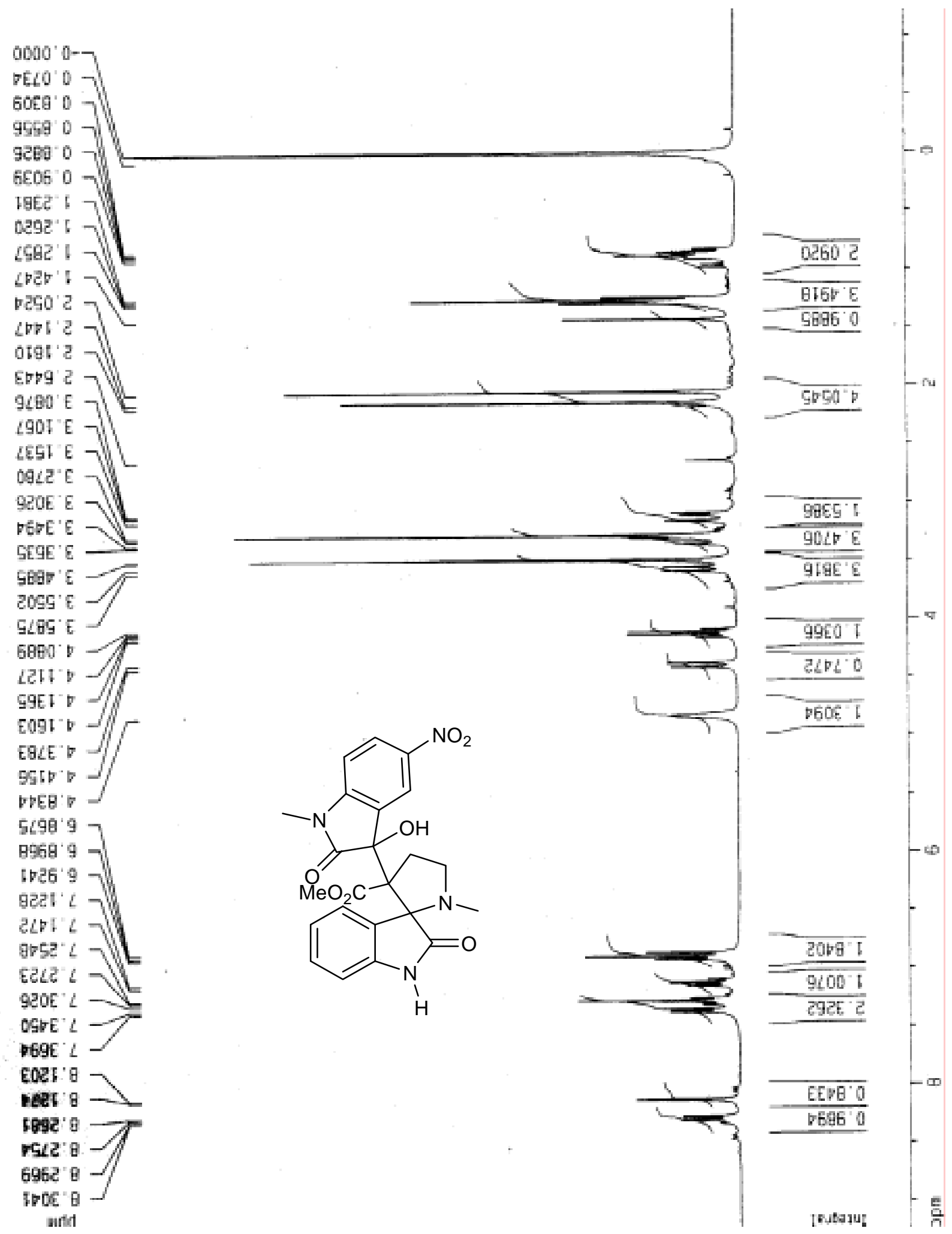




\section{${ }^{1} \mathrm{H}$ NMR spectra of 31}

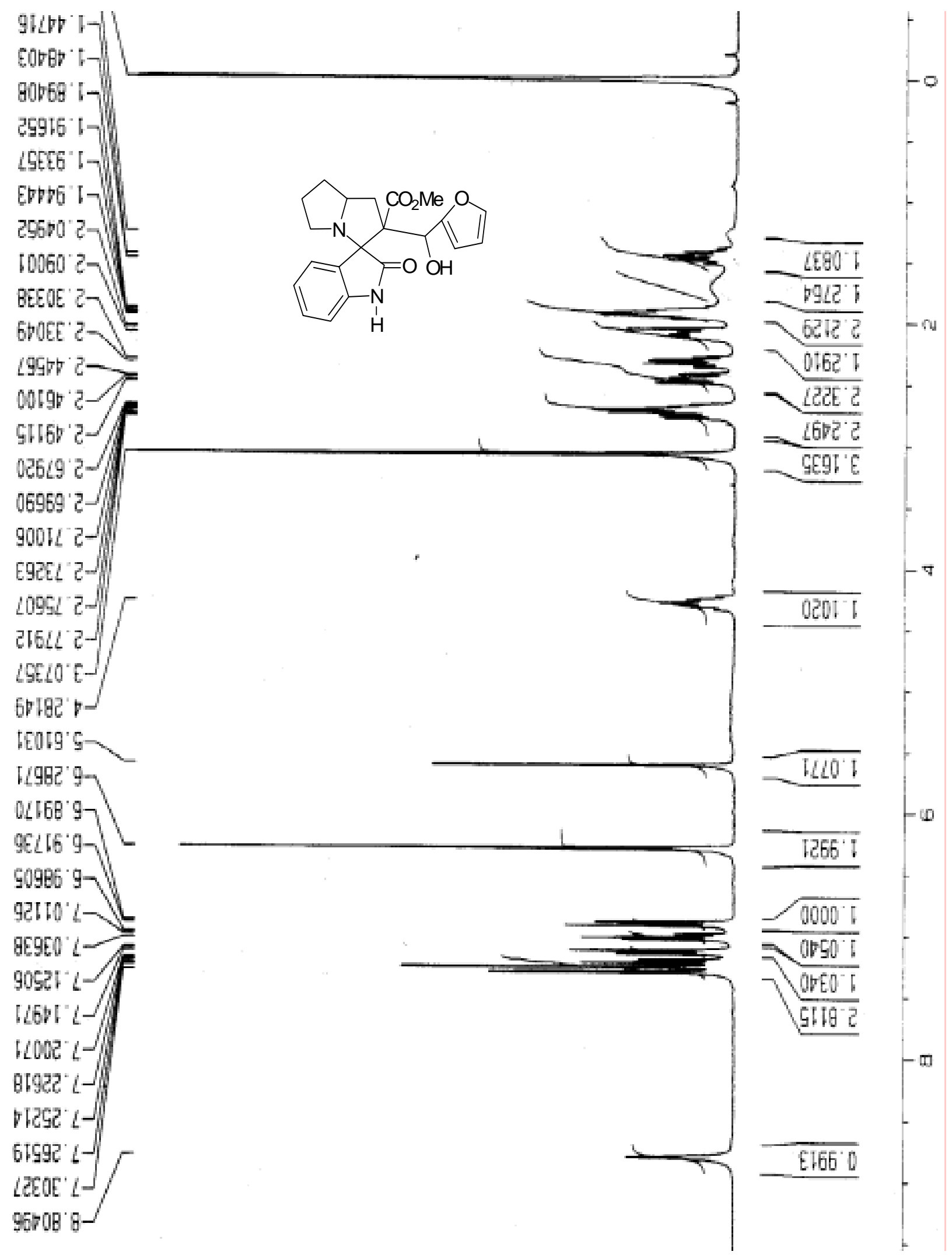


${ }^{13} \mathrm{C}$ NMR spectra of 31
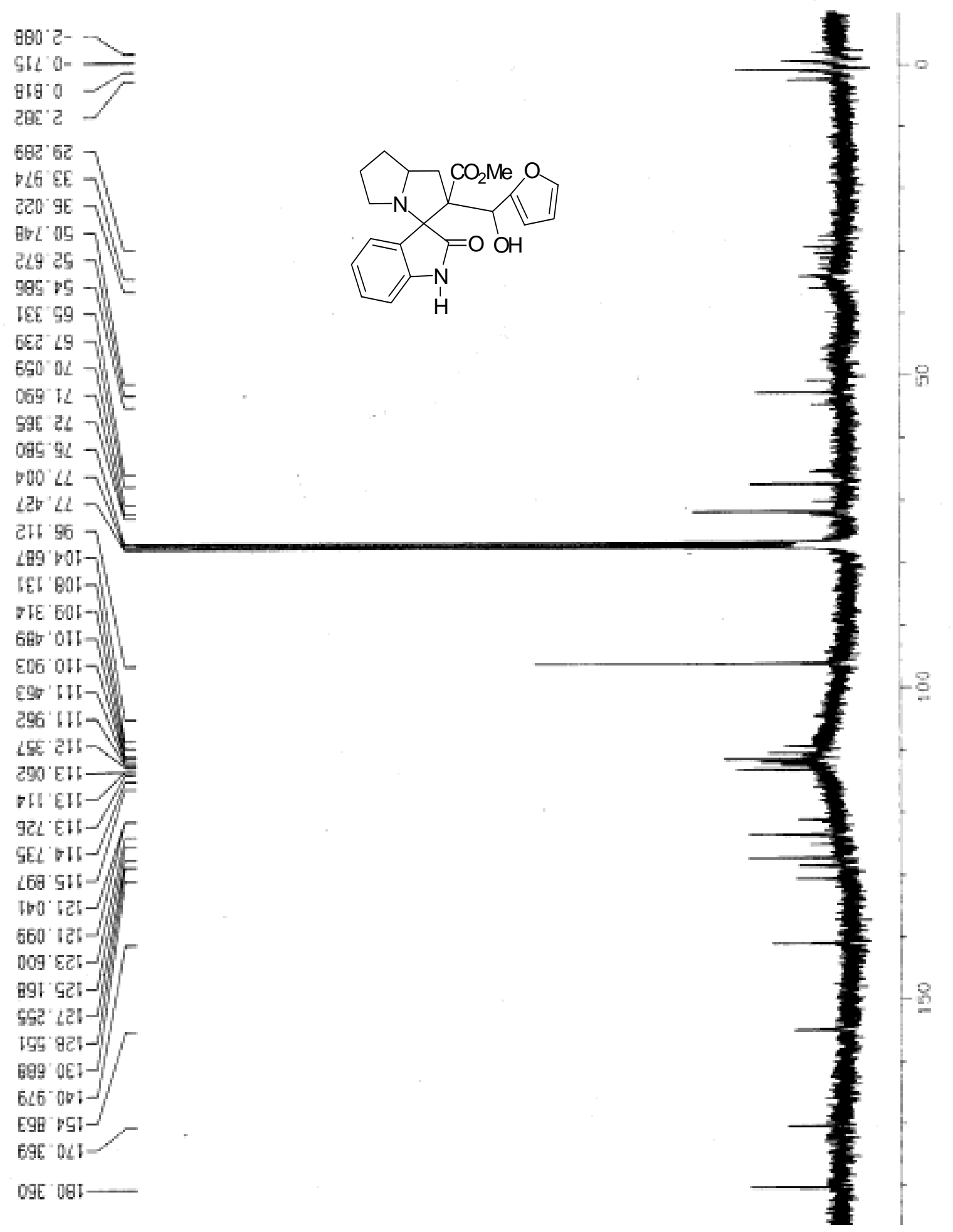


\section{${ }^{1}$ H NMR spectra of 32}

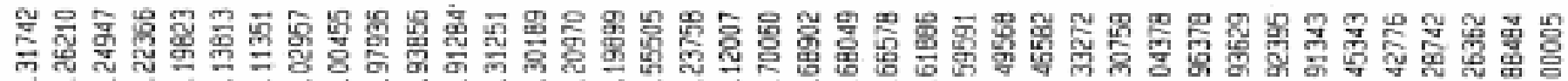

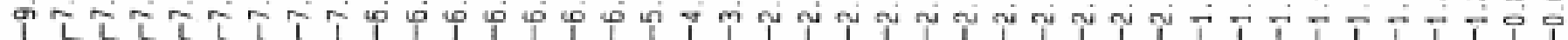<smiles>COC(=O)C1(C(C)=O)CC2CCCN2C12C(=O)Nc1ccccc12</smiles>
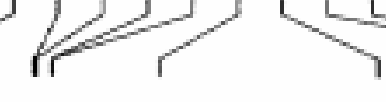


\section{${ }^{13} \mathrm{C}$ NMR spectra of 32}
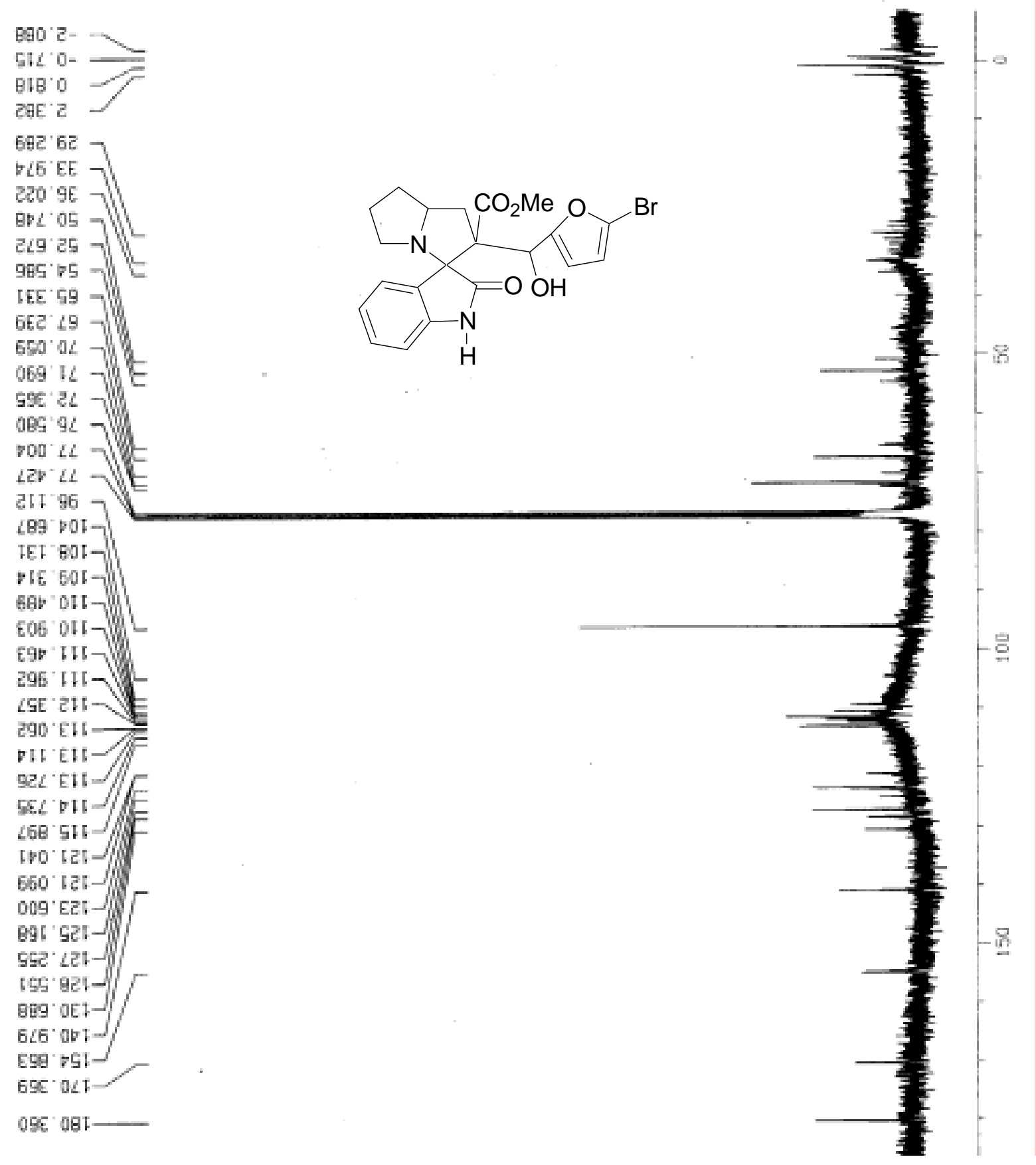
${ }^{1}$ H NMR spectra of 33

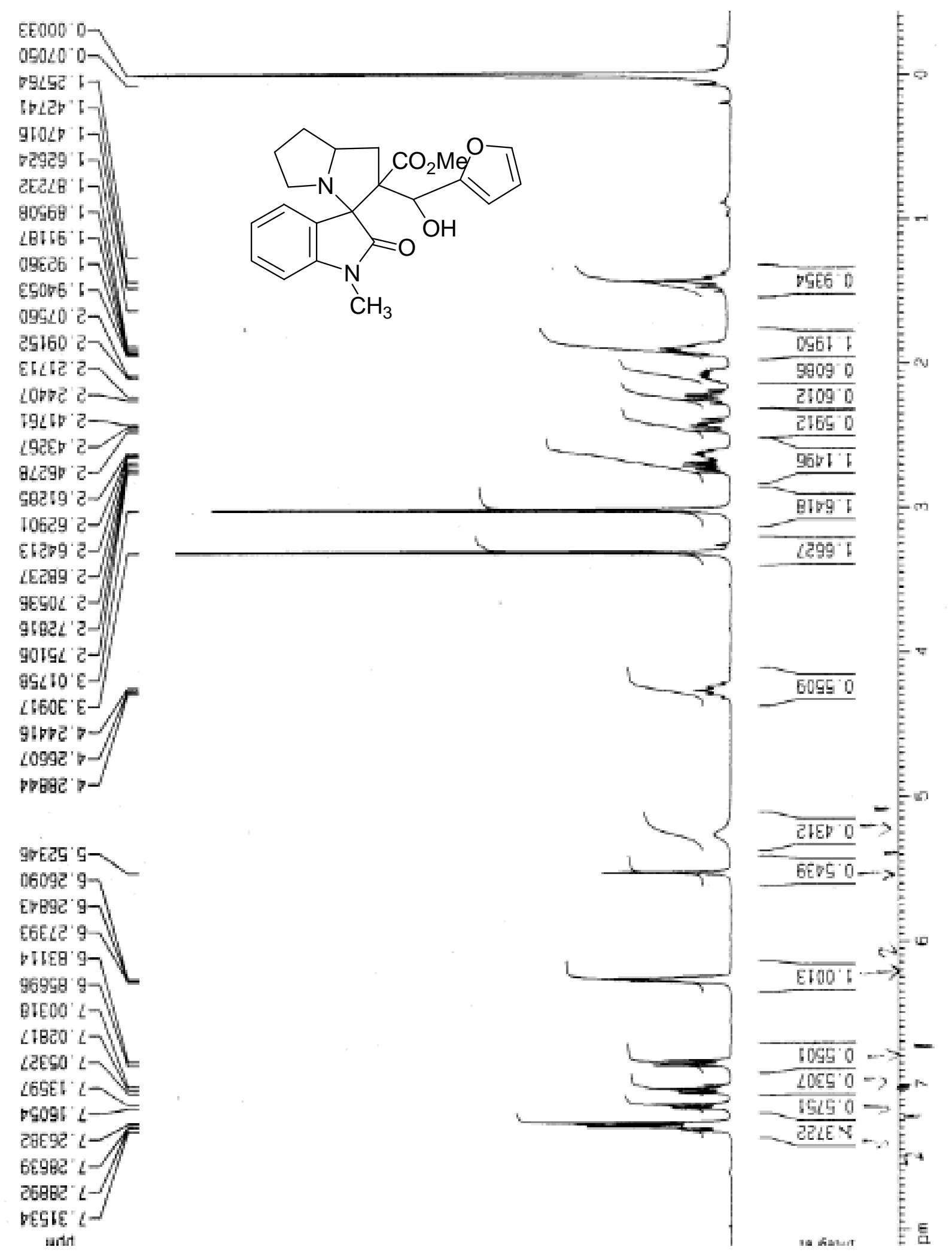


${ }^{13} \mathrm{C}$ NMR spectra of 33
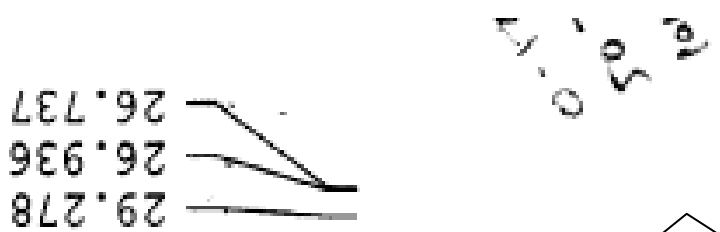

$\varepsilon \varepsilon L^{\circ} \varepsilon \varepsilon$

$\angle 90^{\circ} \nabla \varepsilon$

G $8 \mathrm{I}^{\circ} \nabla \varepsilon$

$9 \mathrm{SO}^{\circ} \mathrm{LV}$

$\varepsilon 6 b^{\circ}$ '
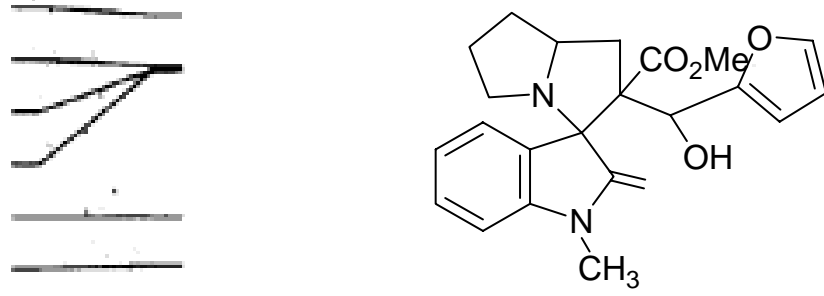

โST. 99

$\varepsilon 6 \tau^{\circ} \tau L$

$966^{\circ} \mathrm{TL}$

万I9.9L

DTL $9 L$

$L E 0^{\circ} L L$

$L E Z^{\circ} L L$

I9 $b^{\circ} L L$
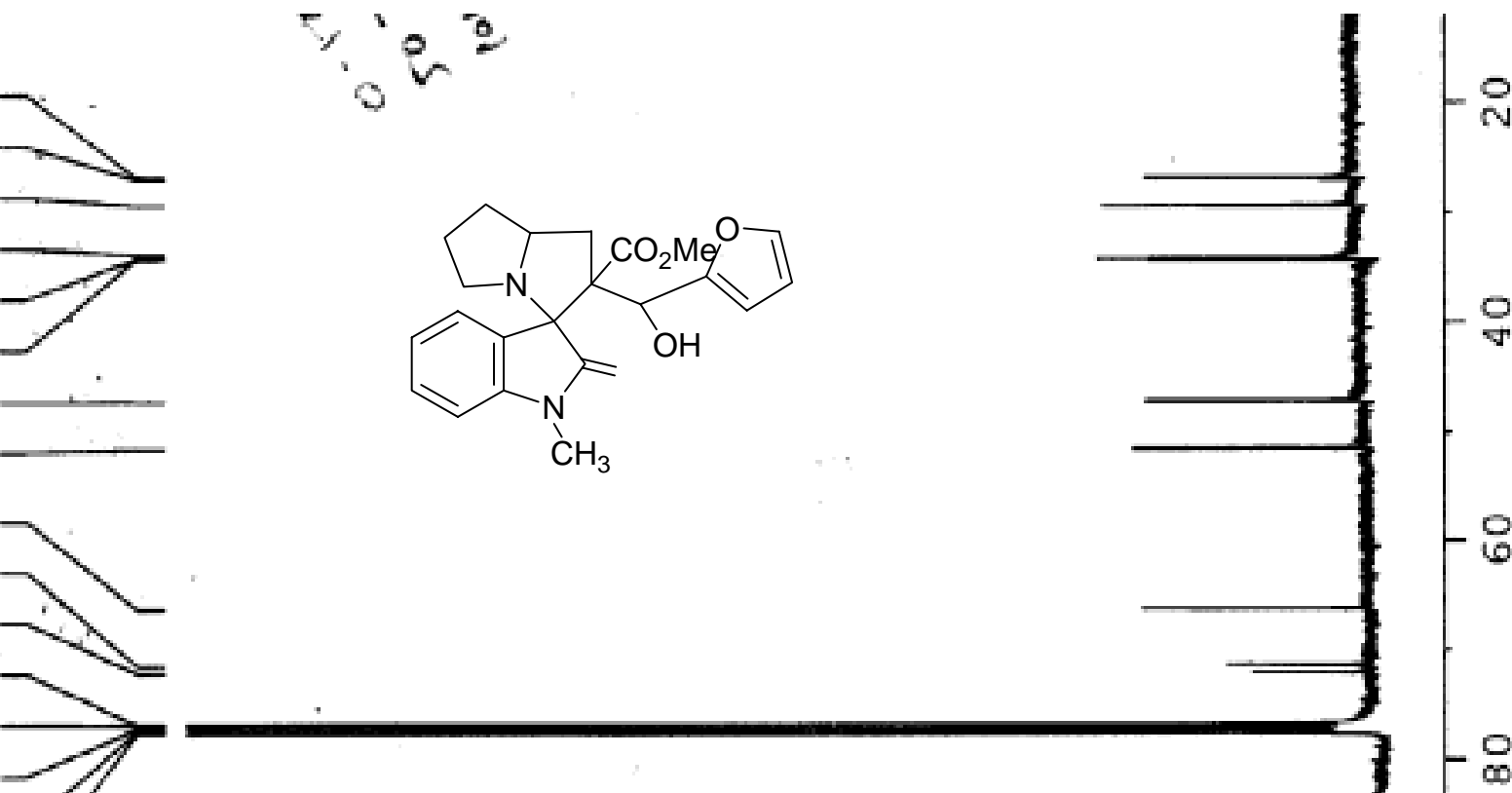

TO ${ }^{\circ}$ LOT

$8 \varepsilon I^{*} 80 \mathrm{~T}$

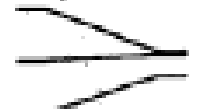

$\angle 86^{\circ} 60 \mathrm{~T}$

I $66^{\circ} \mathrm{ZZT}$

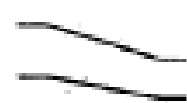

86 I $^{\circ} \mathrm{LZ}$ I

$89 b^{\circ} 62 \mathrm{~T}$

$000^{\circ} Z 6 \mathrm{~T}$

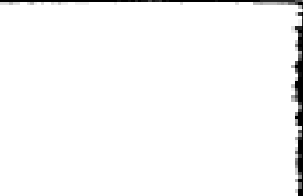

$\infty$

$8 Z 8^{\circ} \varepsilon D T$

$960^{\circ} \varepsilon \varsigma \tau$
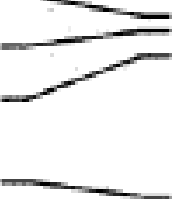
${ }^{1} \mathrm{H}$ NMR spectra of 34

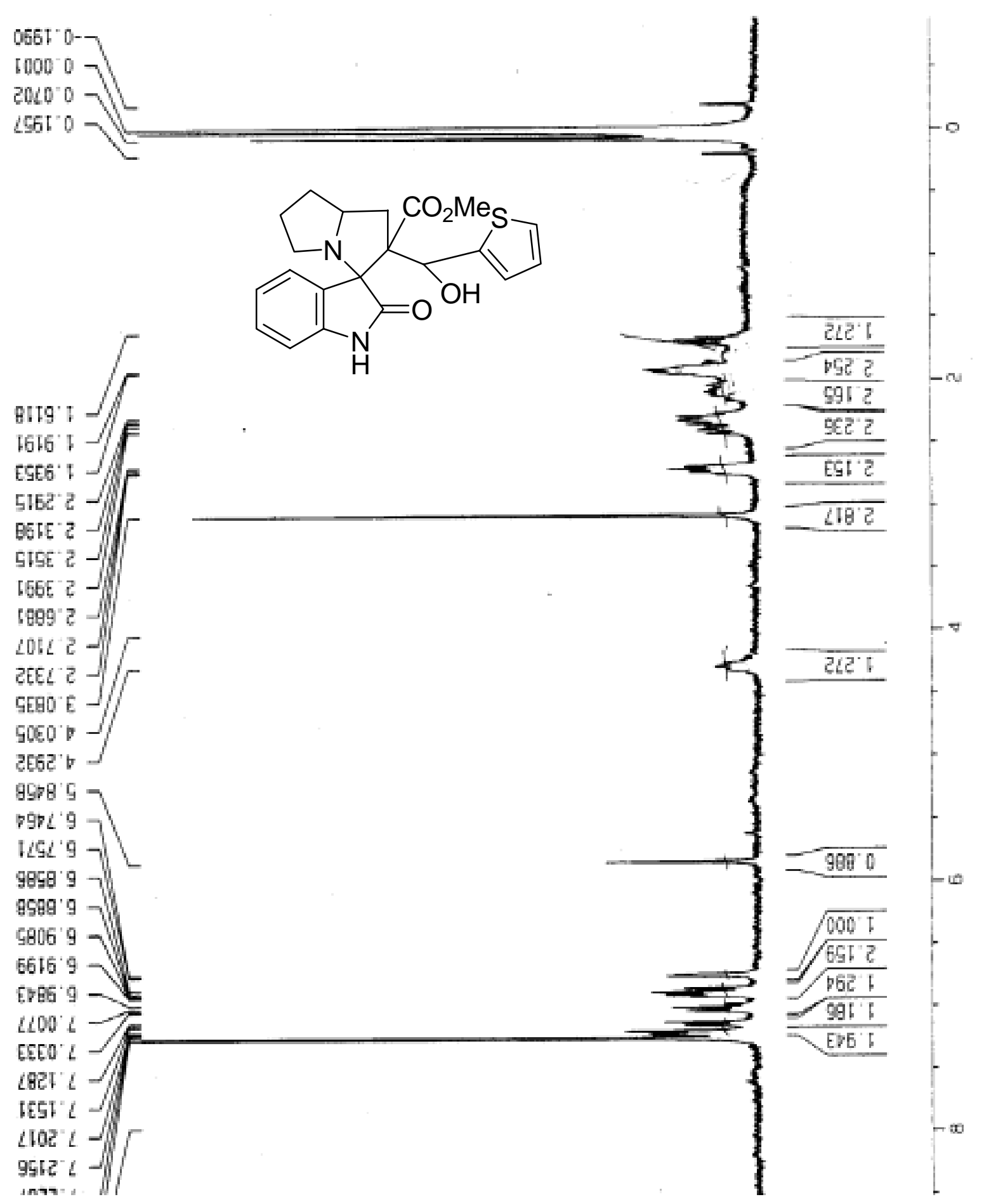




\section{${ }^{1} \mathrm{H}$ NMR spectra of 35}

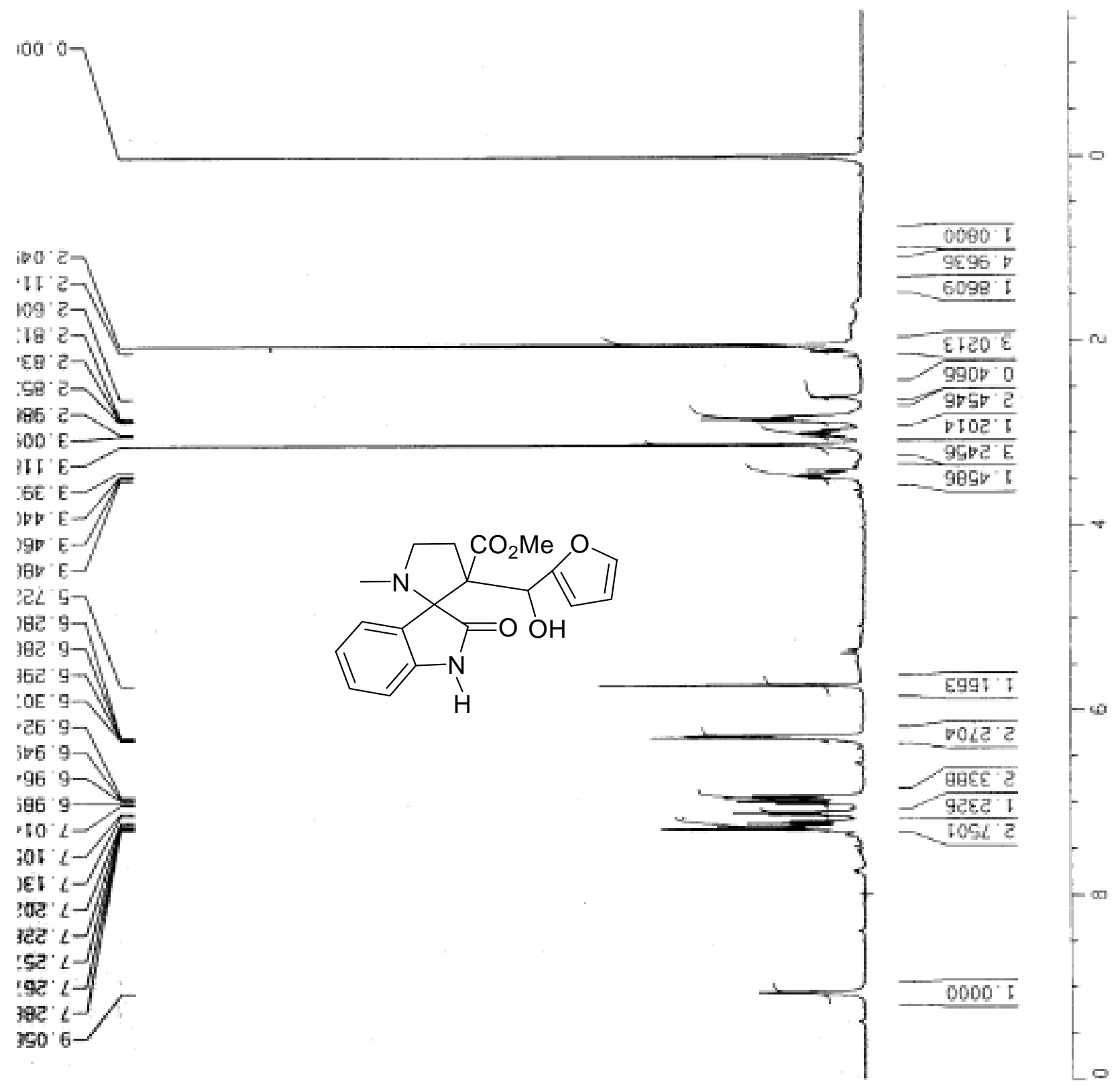


${ }^{13} \mathrm{C}$ NMR spectra of 35
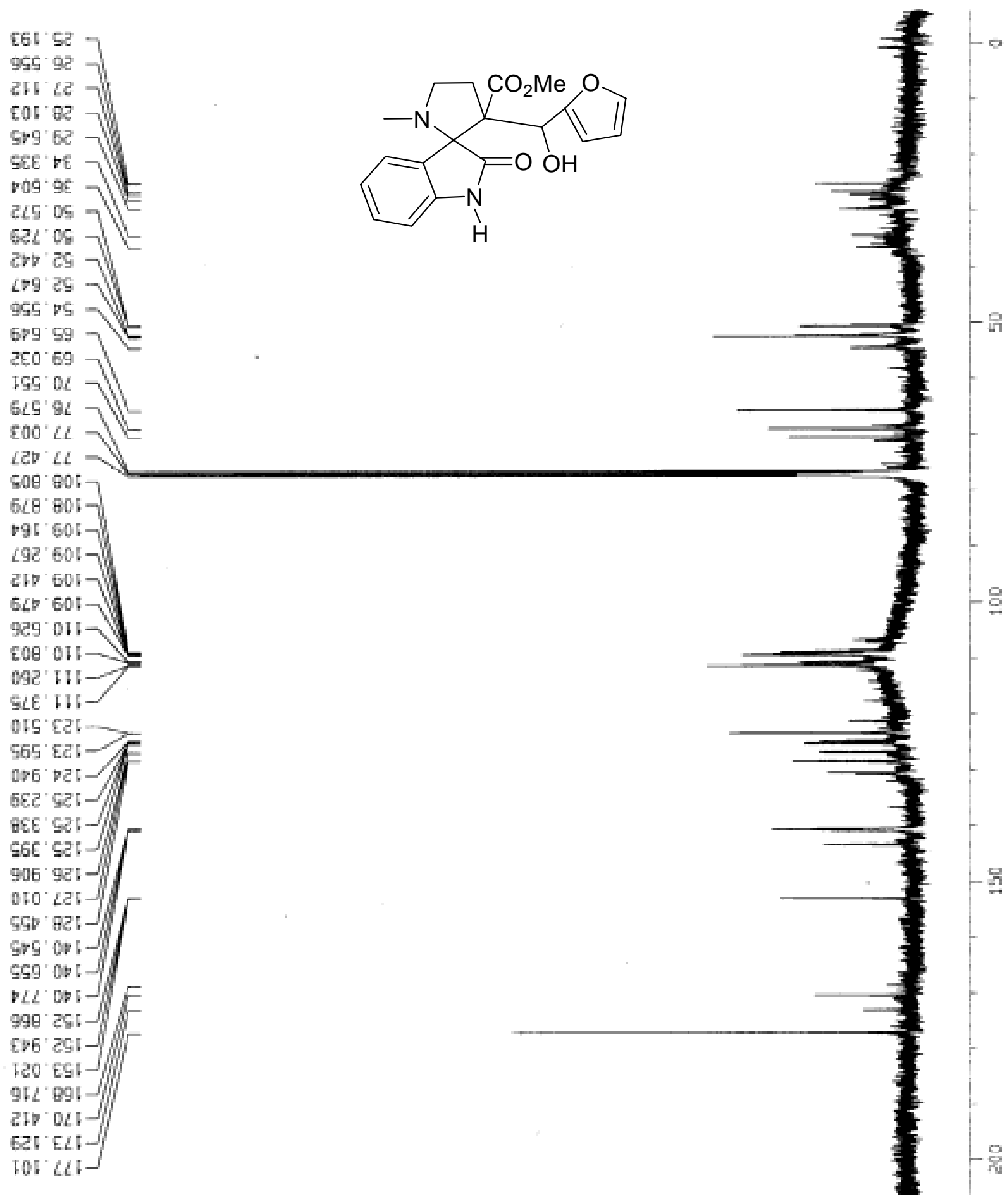


\section{${ }^{1} \mathrm{H}$ NMR spectra of 36}
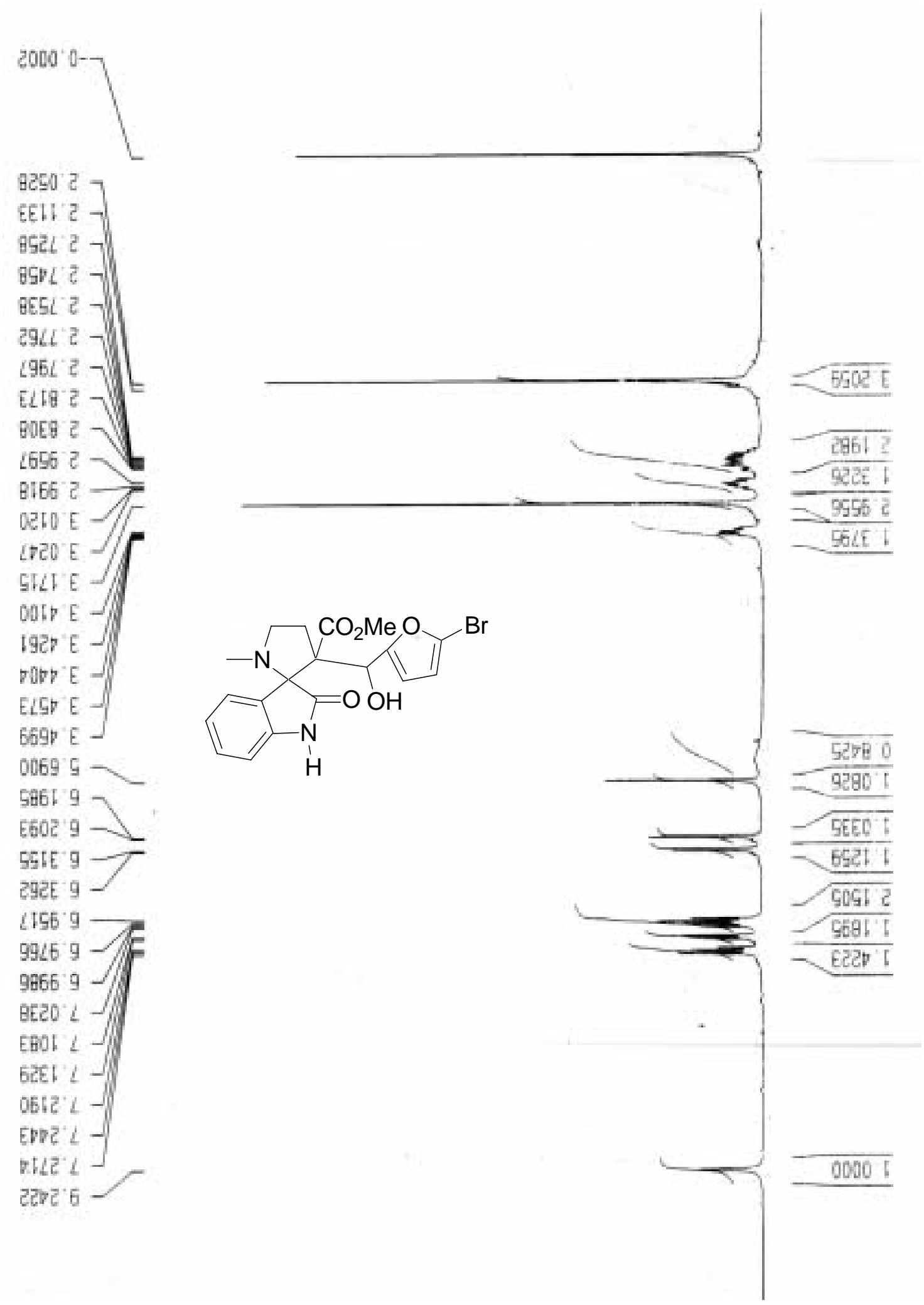
${ }^{1} \mathrm{H}$ NMR spectra of 37

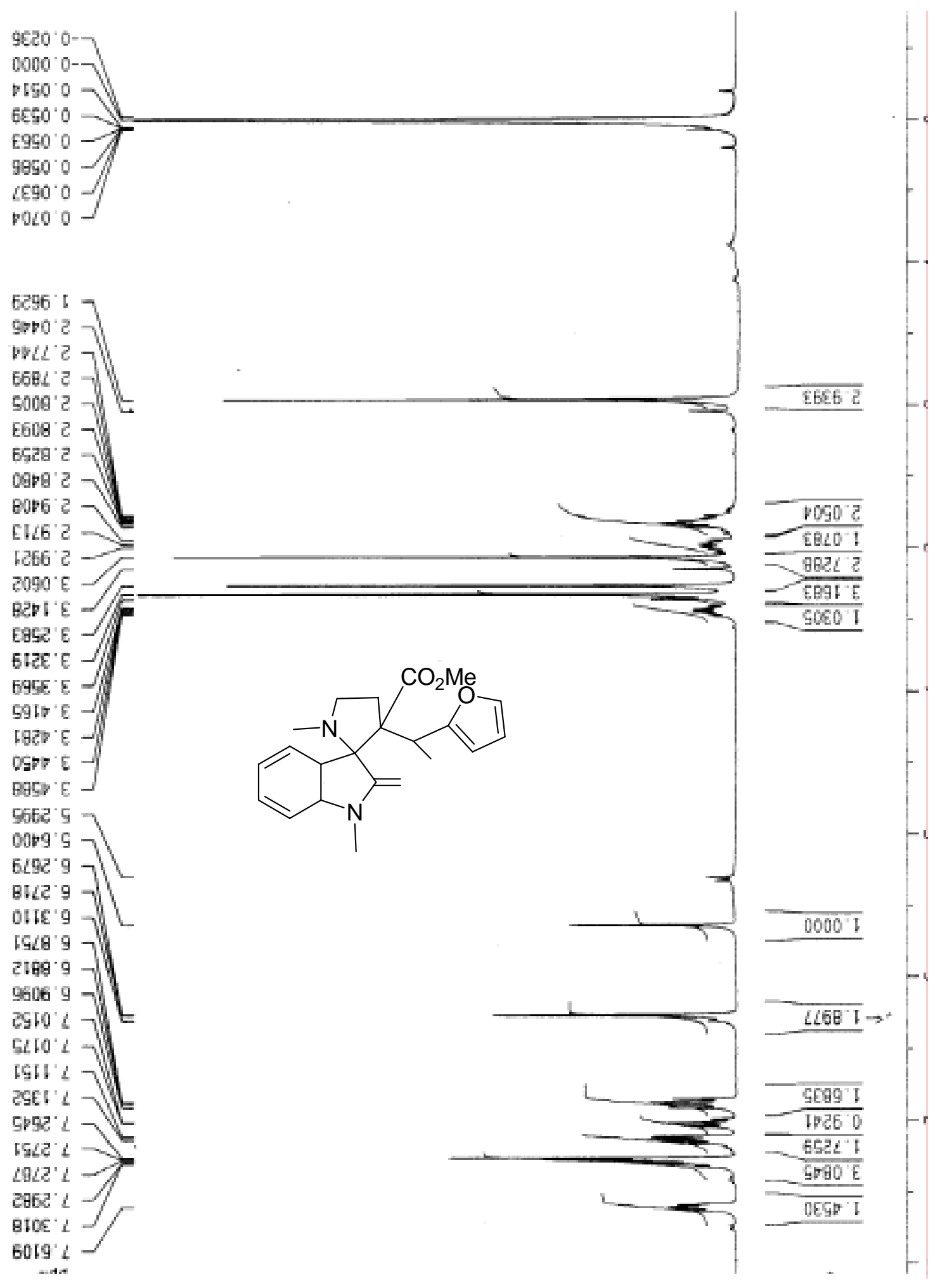


${ }^{1} \mathrm{H}$ NMR spectra of 37

$99 \mathrm{E}^{\circ} \mathrm{E}-$

$\mathrm{ESO}^{\circ} \mathrm{O}-\ldots$

เg8' $ย$ -

เด्व' $0 \mathrm{c}-$

BgS' $\varepsilon$

$9 L 1^{\circ} \mathrm{GC}$

⿰日⿱ 口)

$9 \angle 5 \cdot 92$

TOV GE

GE6 05.

$\checkmark 69^{\prime}$ '

o5p' 25

19999

乌28 69

乌99' $9 L$

$906 \cdot 9 L$

$2 t b^{\circ} L L$

$895^{\circ} \mathrm{COT}$

$600^{\circ} \mathrm{BOS}$

E26.60t-

$6 C 5$ टटा

$\checkmark$ Ig' Eटर

2EC' 525

obg c्रt

$\angle$ टे 6टा

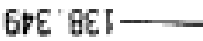

Eto $2 \nabla 5$
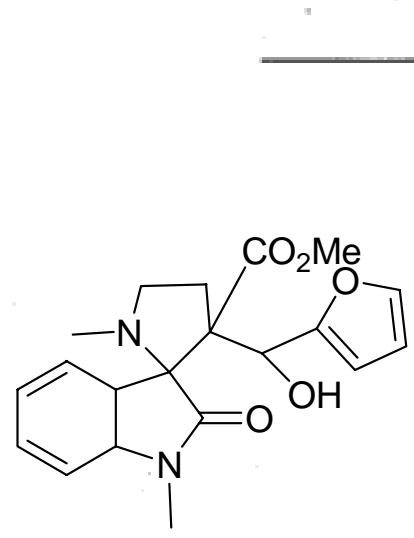


\section{${ }^{1} \mathrm{H}$ NMR spectra of 38}

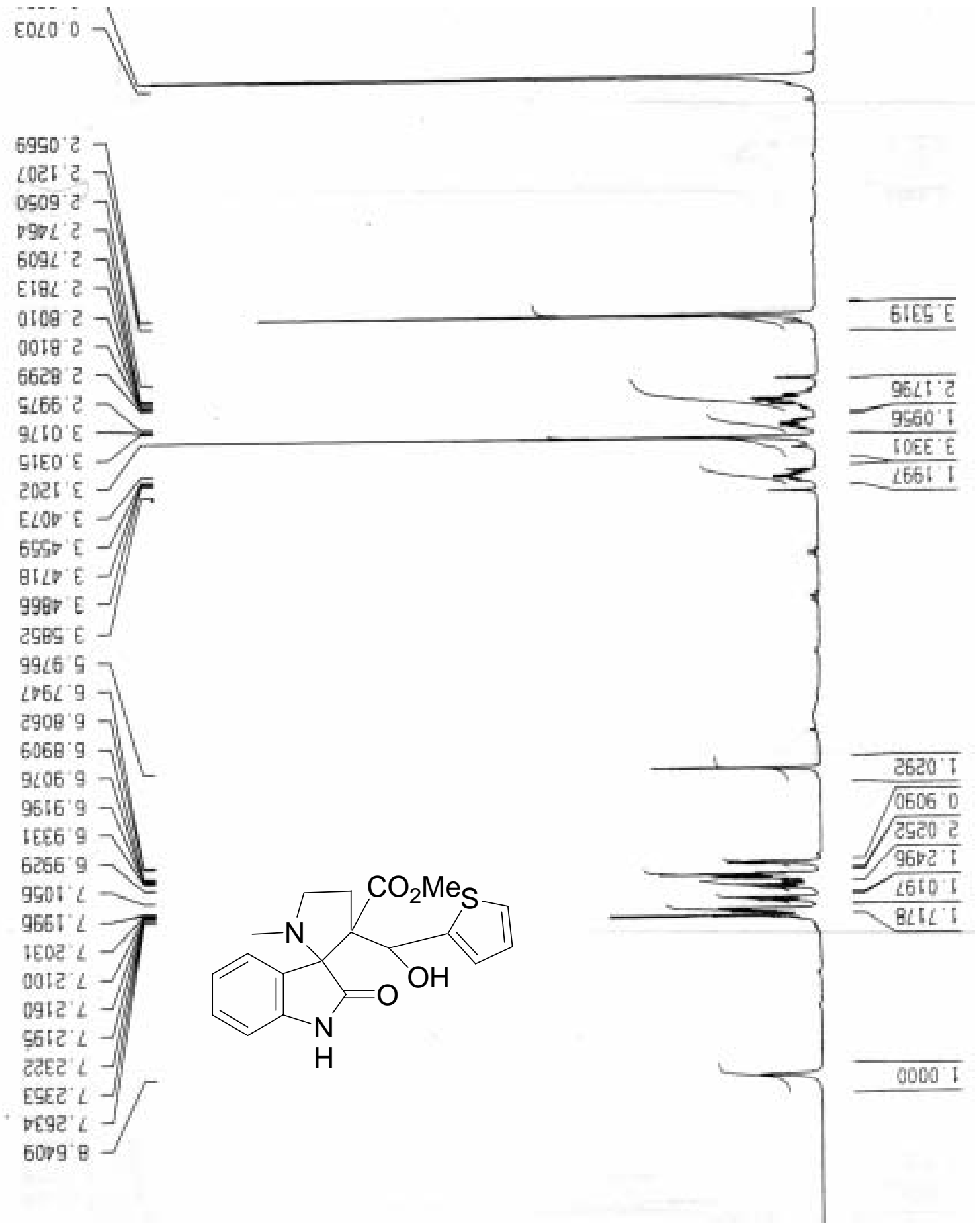




\section{${ }^{13} \mathrm{C}$ NMR spectra of 38}
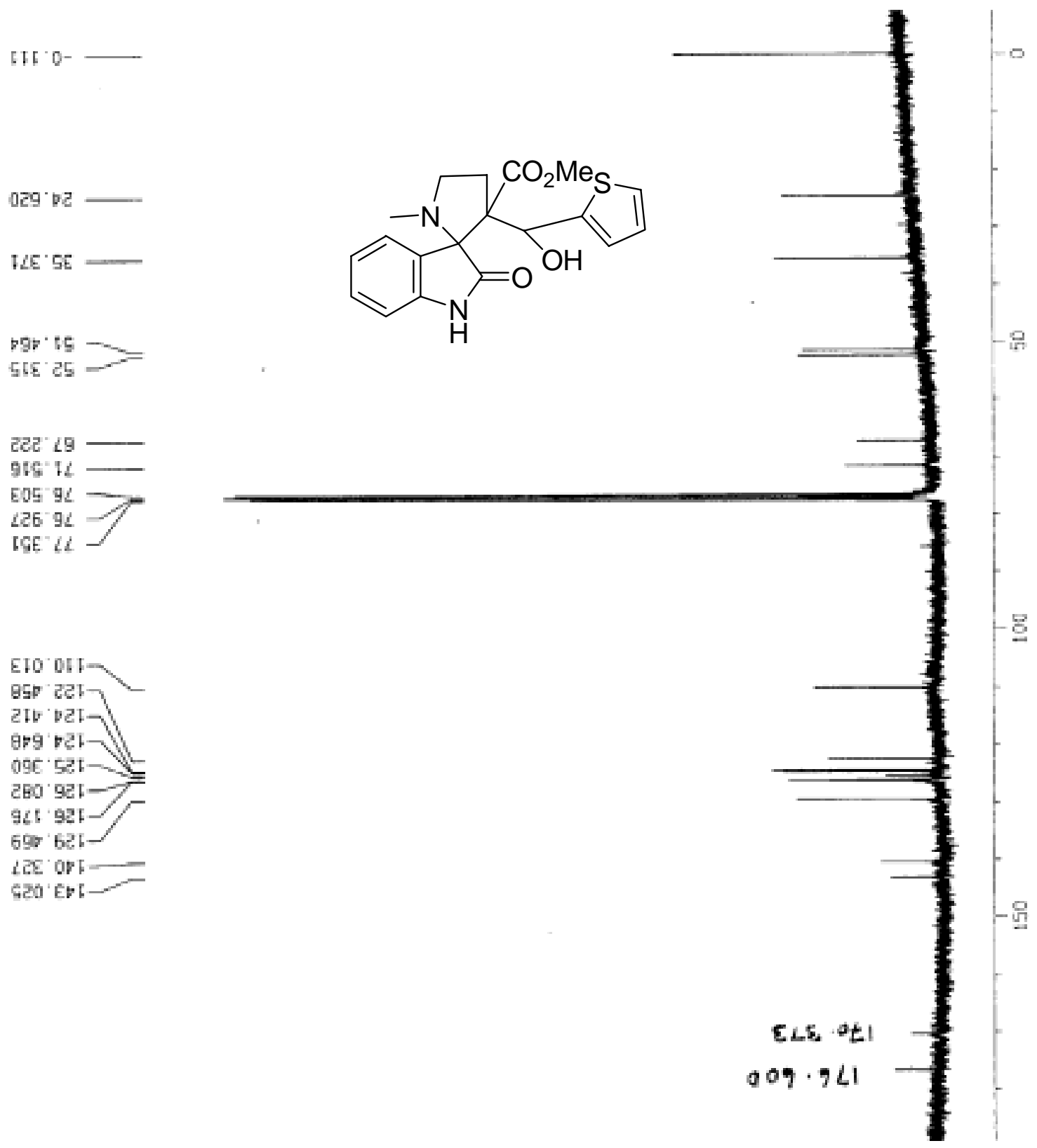